Research Article

\title{
Interval-Valued Hesitant Fuzzy Linguistic Multiattribute Decision-Making Method Based on Three-Parameter Heronian Mean Operators
}

\author{
Wang Juan $\mathbb{C}^{1}$ and Li Qiang $\mathbb{1}^{2}$ \\ ${ }^{1}$ School of Management Science and Engineering, Shandong Technology and Business University, Yantai 264003, China \\ ${ }^{2}$ Department of Safety and Emergency Management, Yantai Engineering and Technology College, Yantai 264006, China \\ Correspondence should be addressed to Wang Juan; 15241887357@163.com
}

Received 6 July 2021; Revised 15 August 2021; Accepted 22 September 2021; Published 19 October 2021

Academic Editor: Feng Feng

Copyright @ 2021 Wang Juan and Li Qiang. This is an open access article distributed under the Creative Commons Attribution License, which permits unrestricted use, distribution, and reproduction in any medium, provided the original work is properly cited.

\begin{abstract}
Numerous variants have been proposed for sets of linguistic terms and the interval-valued hesitant fuzzy set (IVHFS). In particular, the interval-valued hesitant fuzzy linguistic set (IVHFLS) is more suitable for defining the hesitancy and inconsistency inherent in the human cognitive processes of decision making. A key aggregation operator is Heronian mean (HM), based on which the correlation among aggregated arguments can be captured. However, the existing HM operators partially overlook the correlation among more than two arguments and lack the properties of idempotency and reducibility. In this work, the limitations of HM operators are first analyzed. Then, two new HM variants are introduced: three-parameter weighted Heronian mean (TPWHM) and three-parameter weighted geometric Heronian mean (TPWGHM). Thus, the reducibility, idempotency, monotonicity, and boundedness properties are proven for the two computational procedures, and unique situations are mentioned. Furthermore, two more elaborate operators are also introduced which are called the interval-valued hesitant fuzzy linguistic TPWHM (IVHFLTPWHM) and the interval-valued hesitant fuzzy linguistic TPWGHM (IVHFLTPWGHM). The main properties, as well as unique situations of these two computational procedures, are discussed. Finally, the introduced methods are clarified by illustrative examples. In addition, the parameter effects on the decision-making outcomes are discussed and comparisons with other reference methods are made.
\end{abstract}

\section{Introduction}

MADM naturally arises in numerous practical applications, including ones in supplier selection [1], medical diagnosis [2], investment project selection [3], and so on. However, real decision-making problems are complicated by stringent requirements and constraints, including primarily the ability to process fuzzy and vague information. Under these constraints, many methods have been suggested for expressing fuzzy and vague information. Common examples of these methods are those of fuzzy set [4], vague set [5], intuitionistic fuzzy set [6], interval-valued intuitionistic fuzzy set $[7,8]$, and Pythagorean fuzzy set [9].

The traditional methods based on fuzzy sets have been effectively extended by the hesitant fuzzy sets (HFSs)
$[10,11]$, which allow a set of possible membership values. Several HFS variants and enhancements have then emerged. For example, Farhadinia [12, 13] proposed an intervalvalued hesitant fuzzy set (IVHFS) and multiple measuring tools. Arithmetic operations for hesitant fuzzy elements (HFEs) were introduced and several aggregation operators were constructed by Xia et al. [14]. Then, Xu et al. [15] proved key HFE properties and defined corresponding distance and correlation metrics. Further, Peng [16] and Xu [17] investigated some HFS measuring tools. Xu [18] and Akram et al. [19] presented variants of the TOPSIS method within the frameworks of the conventional and neutrosophic hesitant fuzzy sets. Zhu et al. [20] developed the operators of the hesitant fuzzy geometric Bonferroni mean (HFGBM) as well as the hesitant fuzzy Choquet geometric Bonferroni 
mean (HFCGBM). Chen et al. [21] constructed IVHFSs whose membership values could be represented by interval values and hence developed the operator of the intervalvalued hesitant fuzzy weighted averaging (IVHFWA). Wei et al. [22] introduced the operation laws for IVHFS and the scoring function. Peng et al. [23] investigated IVHFS computational procedures to aggregate scores in detail. Naz et al. [24] developed an approach based on interval-valued q-rung orthopair dual hesitant fuzzy set.

For human reasoning, the interval values are generally more appropriate than crisp values for handling fuzzy and imprecise information. Indeed, decision-making problems might sometimes be too vaguely defined or too complex to employ quantitative reasoning. Alternatively, linguistic qualitative variables could be effectively employed in many applications. A linguistic variable was firstly introduced by Zadeh [4] to express qualitative uncertain information. A single or interval-type expression is denoted by a linguistic variable $[25,26]$. Then, HFLSs were investigated by Lin et al. [27] to depict fuzzy object attributes and also indicate potential membership values of a linguistic term. However, under conditions of information fuzziness and complexity, crisp decisions become challenging to make. Interval-type values are generally more suitable for handling information fuzziness and impreciseness. Consequently, IVHFLS was studied by Wang et al. [28] to combine the key advantages of the linguistic approach based on fuzzy concept and IVHFSs. Then, Gong et al. [29] presented an IVHLFS scoring function and proposed a corresponding TOPSIS-based ranking approach. Meng et al. [30] defined the IVHLFS laws of operation and introduced several IVHLFS aggregation operators. Mao et al. [31] addressed MADM problems using cloud computational procedures based on IVHFLNs. Feng et al. [32] proposed a MADM approach with the operator related to the interval-valued q-rung dual hesitant linguistic Maclaurin symmetric mean operators.

Nowadays, the interrelationship among aggregated arguments has been accounted for through the design of novel aggregation operators including the Heronian mean (HM) and the Bonferroni mean (BM) [33, 34]. The HM operators were found to have certain advantages over BM operators, which overlook the operator correlation with attributes $[35,36]$. The HM operators were first introduced by Beliakov et al. [37], who employed the operators for solving MADM problems. Liu et al. [38] proposed a generalized HM (GHM) operator and examined some special cases of this operator. Furthermore, concrete applications of the HM operators have been explored. Yu [39] proposed two operators: the intuitionistic fuzzy geometric HM (IFGHM) as well as the intuitionistic fuzzy geometric weighted HM (IFGWHM). Liu [40] introduced the generalized Heronian OWA operator, which shares similar characteristics with the BM and Bonferroni OWA operators. Liu et al. [41] developed a MAGDM algorithm utilizing the operator based on the 2tuple linguistic neutrosophic Dombi power HM. Xu et al. [42] implemented the copula HM when a linguistic intervalvalued intuitionistic fuzzy decision making is a concern. Ayub et al. [43] constructed several cubic fuzzy HM Dombi aggregation operators, which were based on combining the Dombi operational laws and HM operators. Lin et al. [44] presented a MADM algorithm with picture fuzzy interactional partitioned HM aggregation operators. Tian et al. [45] developed an MCDM method with the partitioned HM (PHM) operator and the Shapley fuzzy measure with a single-valued neutrosophic set.

Although significant progress has been made in the theory and applications of the HM, HFS, HFLS, and IVHFLS operators, some shortcomings persist. (1) The existing HM operators consider only the correlation among pairs of aggregated arguments but ignore higher-order complex correlation patterns among three or more aggregated arguments. (2) The generalized HM (GHM) and weighted geometric HM (WGHM) operators do not satisfy the properties of reducibility and idempotency and hence cannot be effectively employed in addressing real decisionmaking problems. Thus, in our work, we have two essential contributions. Firstly, we introduce two novel HM operators, namely, the three-parameter weighted HM (TPWHM) and the three-parameter weighted geometric HM (TPWGHM), which are shown to be reducible, idempotent, monotone, and bounded and they do not suffer from deficiencies that are already mentioned. The other sections of the manuscript are organized as follows. Key concepts and operational laws for interval numbers, IVHFSs, and (IVHFLSs) are reviewed in Section 2. In Section 3, we equip the HM operators with three parameters and propose the three-parameter weighted HM (TPWHM) and the three-parameter weighted geometric HM (TPWGHM) operators. Section 4 deals with the TPWHM and TPWGHM operators which are extended to aggregate IVHFL information. Section 5 gives computational tools for the decision-making procedure. Section 6 points outs an example based on our proposed method and demonstrates the advantages of our approach through comparisons and sensitivity analysis. Section 7 concludes the research.

\section{Preliminaries}

Here, a brief review is presented containing basic concepts and operations for interval numbers, IVHF numbers, and FL numbers.

\subsection{Interval Numbers}

Definition 1 (see [46]). $\widetilde{a}$ denotes an interval number, i.e., $\tilde{a}=\left[a^{L}, a^{U}\right]$ or $\tilde{a}=\left\{x \mid a^{L} \leq x \leq a^{U}\right\}$ where the lower and upper endpoints of interval number are denoted by $a^{\mathrm{L}}$ and $a^{U}$ in the support of $\widetilde{a}$, respectively. Two intervals are denoted by $\widetilde{a}=\left[a^{L}, a^{U}\right]$ and $\widetilde{b}=\left[b^{L}, b^{U}\right]$. For these numbers, the arithmetic of interval numbers is expressed by
(1) $\tilde{a} \oplus \widetilde{b}=\left[a^{L}+b^{L}, a^{U}+b^{U}\right]$.
(2) $\tilde{a} \otimes \widetilde{b}=\left[a^{L} b^{L}, a^{U} b^{U}\right]$.
(3) $\lambda \otimes \widetilde{a}=\left[\lambda a^{L}, \lambda a^{U}\right], \lambda \geq 0$.
(4) $a^{\lambda}=\left[\left(a^{L}\right)^{\lambda},\left(a^{U}\right)^{\lambda}\right], \lambda \geq 0$. 
Definition 2 (see [46]). Two intervals are denoted by $\widetilde{a}=$ $\left[a^{L}, a^{U}\right]$ and $\tilde{b}=\left[b^{L}, b^{U}\right]$. Then, len $(\tilde{a})=a^{U}-a^{L}$ and len $(\tilde{b})=b^{U}-b^{L}$ are called the lengths of those intervals. The possibility degree for $\widetilde{a} \geq \widetilde{b}$ is given as follows:

$$
p(\widetilde{a} \geq \widetilde{b})=\max \left\{1-\max \left[\frac{a^{U}-b^{L}}{\operatorname{len}(\widetilde{a})+\operatorname{len}(\widetilde{b})}, 0\right], 0\right\} .
$$

Likewise, the possibility degree that $\tilde{b} \geq \tilde{a}$ is given as follows:

$$
p(\widetilde{b} \geq \widetilde{a})=\max \left\{1-\max \left[\frac{a^{\mathrm{U}}-b^{\mathrm{L}}}{\operatorname{len}(\widetilde{a})+\operatorname{len}(\widetilde{b})}, 0\right], 0\right\} .
$$

\subsection{IVHFS}

Definition 3 (see [46]). A nonempty fixed set is denoted by $X$. The HFS $H X$ is defined as follows:

$$
H=\left\{\left\langle x, \widetilde{h}_{H}(x)\right\rangle \mid x \in X\right\}
$$

where the function $\tilde{h}_{H}(x): X$ returns a subset of $[0,1]$, which represents the possible membership degrees of a given element $x \in X$ in $H$. The basic HFS unit is $h=\widetilde{h}_{H}(x)$.

Definition 4 (see [47]). A nonempty fixed set is denoted by $\mathrm{X}$. IVHFS $E$ on $X$ is

$$
E=\left\{\left\langle x, \tilde{h}_{E}(x)\right\rangle \mid x \in X\right\},
$$

where $\tilde{h}_{E}(x): X$ is the set containing all possible interval fuzzy values in $[0,1]$, which extracts the possible degrees of the membership of the element $x \in X$ in $E . \widetilde{h}=\widetilde{h}_{E}\left(x_{i}\right)=$ $\left\{\tilde{\gamma}=\left[\gamma^{L}, \gamma^{U}\right] \mid \tilde{\gamma} \in \widetilde{h}_{E}\left(x_{i}\right)\right\}$ is called an IVHFE.

Definition 5 (see [46]). Let $\tilde{h}=\left\{\left[\gamma^{L}, \gamma^{U}\right]\right\} \tilde{h}_{1}=\left\{\left[\gamma_{1}^{L}, \gamma_{1}^{U}\right]\right\}$ and $\widetilde{h}_{2}=\left\{\left[\gamma_{2}^{L}, \gamma_{2}^{U}\right]\right\}$ be three IVHFEs.

The IVHFE operational rules are

(1) $\tilde{h}^{\lambda}=\cup_{\tilde{\gamma} \in \tilde{h}}\left\{\left[\left(\gamma^{L}\right)^{\lambda},\left(\gamma^{U}\right)^{\lambda}\right]\right\}$.

(2) $\lambda \tilde{h}=\cup_{\tilde{\gamma} \in \tilde{h}}\left\{\left[1-\left(1-\gamma^{L}\right)^{\lambda}, 1-\left(1-\gamma^{U}\right)^{\lambda}\right]\right\}, \lambda>0$.

(3) $\begin{aligned} & \tilde{h}_{1} \oplus \widetilde{h}_{2}=\cup_{\tilde{\gamma}_{1} \in \tilde{h}_{1}, \tilde{\gamma}_{2} \in \tilde{h}_{2}}\left\{\left[\gamma_{1}^{L}+\gamma_{2}^{L}-\gamma_{1}^{L} \gamma_{2}^{L}, \gamma_{1}^{U}+\gamma_{2}^{U}-\gamma_{1}^{U}\right.\right. \\ & \left.\left.\gamma_{2}^{U}\right]\right\} .\end{aligned}$

(4) $\tilde{h}_{1} \otimes \tilde{h}_{2}=\cup_{\tilde{\gamma}_{1} \in \tilde{h}_{1}, \tilde{\gamma}_{2} \in \tilde{h}_{2}}\left\{\left[\gamma_{1}^{L} \gamma_{2}^{L}, \gamma_{1}^{U} \gamma_{2}^{U}\right]\right\}$.

Definition 6 (see [47])). The scoring function for any IVHFE $\widetilde{h}$ is

$$
s(\widetilde{h})=\frac{1}{\# \widetilde{h}} \sum_{\tilde{\gamma} \in \widetilde{h}} \widetilde{\gamma}
$$

where $\# \widetilde{h}$ is the number of interval values in $\tilde{h}$. Obviously, $s(\widetilde{h})$ is an interval value belonging to $[0,1]$.

Let $\widetilde{h}_{1}$ and $\widetilde{h}_{2}$ be any two IVHFEs. The IVHFE comparison laws are as follows:

(1) If $s\left(\widetilde{h}_{1}\right) \geq s\left(\widetilde{h}_{2}\right)$, then $\widetilde{h}_{1} \geq \widetilde{h}_{2}$.

(2) If $s\left(\widetilde{h}_{1}\right)<s\left(\widetilde{h}_{2}\right)$, then $\widetilde{h}_{1}<\widetilde{h}_{2}$.
Two score functions can be compared using equation (5), while the magnitude of two IVHFEs can be evaluated based on Definition 2.

2.3. IVHFLSs. IVHFLSs are defined based on linguistic sets and IVHFSs.

Definition 7 (see [28]). Let $x=\left\{x_{1}, x_{2}, \cdots, x_{n}\right\}$ denote a reference set $s_{\theta(x)} \in S, \theta(x) \in\{0,1,2, \ldots, 2 t\}$. The IVHFLS is defined by

$$
A=\left\{\left\langle x, s_{\theta(x)}, \Gamma_{A(x)}\right\rangle, x \in X\right\},
$$

where $\Gamma_{A(x)}$ denotes a set of finite closed intervals of $[0,1]$ :

$$
\Gamma_{A(x)}=\left\{\left[a_{i}^{-}, a_{i}^{+}\right], i=1,2, \ldots, \# \Gamma_{A(x)}\right\},
$$

where the count of the interval values of $A$ is expressed by $\# \Gamma_{A(x)}$. We call $\left\langle x, s_{\theta(x)}, \Gamma_{A(x)}\right\rangle$ an interval-valued hesitant fuzzy linguistic element (IVHFLE).

Likewise, utilizing the IVHFLE and HFLE computational procedures, basic IVHFLE operational laws are given next.

Definition 8 Let $a=\left\langle s_{\theta(a)}, \Gamma_{\theta(a)}\right\rangle, a_{1}=\left\langle s_{\theta\left(a_{1}\right)}, \Gamma_{\theta\left(a_{1}\right)}\right\rangle$, and $a_{2}=\left\langle s_{\theta\left(a_{2}\right)}, \Gamma_{\theta\left(a_{2}\right)}\right\rangle$ be three IVHFLEs. Then, $\gamma_{\theta(a)}=$ $\left[\gamma_{\theta(a)}^{L}, \gamma_{\theta(a)}^{U}\right] \in \Gamma_{\theta(a)}, \gamma_{\theta\left(a_{1}\right)}=\left[\gamma_{a_{1}}^{\mathrm{L}}, \gamma_{a_{1}}^{\mathrm{U}}\right] \in \Gamma_{\theta\left(a_{1}\right)}, \gamma_{\theta\left(a_{2}\right)}=\left[\gamma_{a_{2}}^{L}\right.$, $\left.\gamma_{a_{2}}^{U}\right] \in \Gamma_{\theta\left(a_{2}\right)}$. The operations on these elements are

(1) $\operatorname{neg}(a)=\left\langle s_{2 t-\theta(a)}, \cup_{\gamma_{\theta a} \in \Gamma_{\theta(a)}}\left\{\left[1-\gamma_{a}^{L}, 1-\gamma_{a}^{U}\right]\right\}\right.$.

(2) $a_{1} \oplus a_{2}=\left\langle s_{\theta\left(a_{1}\right)+\theta\left(a_{2}\right)}, \bigcup \gamma_{\theta\left(a_{1}\right)} \in \Gamma_{\theta\left(a_{1}\right)}, \gamma_{\theta\left(a_{2}\right)} \in \Gamma_{\theta\left(a_{2}\right)}\right.$ $\left.\left\{\left[\gamma_{a_{1}}^{\mathrm{L}}+\gamma_{a_{2}}^{\mathrm{L}}-\gamma_{a_{1}}^{\mathrm{L}} \gamma_{a_{2}}^{\mathrm{L}}, \gamma_{a_{1}}^{\mathrm{U}}+\gamma_{a_{2}}^{\mathrm{U}}-\gamma_{a_{1}}^{\mathrm{U}} \gamma_{a_{2}}^{\mathrm{U}}\right]\right\}\right\rangle$

(3) $a_{1} \otimes a_{2}=\left\langle s_{\theta\left(a_{1}\right) \otimes \theta\left(a_{2}\right)}, \cup_{\gamma_{\theta\left(a_{1}\right)} \in \Gamma_{\theta\left(a_{1}\right)}, \gamma_{\theta\left(a_{2}\right)} \in \Gamma_{\theta\left(a_{2}\right)}}\left\{\left[\gamma_{a_{1}}^{\mathrm{L}} \gamma_{a_{2}}^{\mathrm{L}}\right.\right.\right.$, $\left.\left.\left.\gamma_{a_{1}}^{\mathrm{U}} \gamma_{a_{2}}^{\mathrm{U}}\right]\right\}\right\rangle$.

(4) $a^{\lambda}=\left\langle s_{\theta(a)^{\lambda}}, \cup_{\gamma_{\theta a} \in \Gamma_{\theta(a)}}\left\{\left[\left(\gamma_{a}^{L}\right)^{\lambda},\left(\gamma_{a}^{U}\right)^{\lambda}\right]\right\}\right.$.

(5) $\lambda a=\left\langle s_{\lambda \theta(a)}, \cup_{\gamma_{\theta a} \in \Gamma_{\theta(a)}}\left\{\left[1-\left(1-\gamma_{a}^{L}\right)^{\lambda}, 1-\left(1-\gamma_{a}^{U}\right)^{\lambda}\right]\right\}\right\rangle$, $\lambda>0$.

Definition 9 (see [27]). The scoring function for any IVHFLE $\mathrm{a}$ is

$$
s(a)=\left(\frac{1}{2 \# \Gamma_{\theta(a)}} \sum_{\gamma_{\theta(a)} \in \Gamma_{\theta(a)}} \frac{1}{\gamma}\right)
$$

where the count of the interval values $\tilde{h}$ is denoted by $\# \Gamma_{\theta(a)}$ and $s(a)$ is an interval value in $[0,1]$.

\section{Three-Parameter Heronian Mean (TPHM) Operators}

The HM is a key aggregation operator, through which the relations among individual arguments can be captured. Compared with other aggregation operators, the HM operator can more effectively capture the correlation of aggregated arguments. However, existing HM operators consider only pairwise correlation among arguments and also do not satisfy the idempotency and reducibility 
properties. To overcome these limitations, we propose the operators of the three-parameter weighted HM (TPWHM). Ternary interconnections between arguments could be captured by new operators, and also some desirable properties could be demonstrated.

3.1. Heronian Mean. The Heronian mean (HM) is an essential aggregation operator for capturing pairwise argument correlation [26, 28, 29]. This operator can be mathematically defined as follows.

Definition 10 (see [38]). A nonnegative number set is denoted by $c_{i}(i=1,2, \ldots, n)$. HM is expressed by

$$
\operatorname{HM}\left(c_{1}, c_{2}, \ldots, c_{n}\right)=\frac{2}{n(n+1)} \sum_{i=1}^{n} \sum_{j=i}^{n} \sqrt{c_{i} c_{j}} .
$$

The idempotency, monotonicity, and boundedness properties hold for this operator.
Definition 11 (see [38]). A nonnegative number set is denoted by $c_{i}(i=1,2, \ldots, n)$. For $p, q \geq 0$, the generalized $\mathrm{HM}(\mathrm{GHM})$ is expressed by

$$
\operatorname{GHM}^{p, q}\left(c_{1}, c_{2}, \ldots, c_{n}\right)=\left(\frac{2}{n(n+1)} \sum_{i=1}^{n} \sum_{j=1}^{n} c_{i}^{p} c_{j}^{q}\right)^{1 / p+q} .
$$

The idempotency, monotonicity, and boundedness properties again hold for this operator [28].

Definition 12 (see [36]). Let $\left\{c_{1}, c_{2}, \ldots, c_{n}\right\}$ be a set of nonnegative numbers. Also, a weight vector $w=\left(w_{1}, w_{2}, \ldots, w_{n}\right)^{T}$ is assumed such that $w_{i}>0$ and $\sum_{i=1}^{n} w_{i}=1$. For $p, q \geq 0$, the generalized weighted HM (GWHM) operator is

$$
\operatorname{GWHM}^{p, q}\left(c_{1}, c_{2}, \ldots, c_{n}\right)=\left(\frac{2}{n(n+1)} \sum_{i=1}^{n} \sum_{j=i}^{n}\left(w_{i} c_{i}\right)^{p}\left(w_{j} c_{j}\right)^{q}\right)^{1 / p+q}
$$

Definition 13 (see [39]). Let $\left\{c_{1}, c_{2}, \ldots, c_{n}\right\}$ be a set of $n$ nonnegative numbers. $w=\left(w_{1}, w_{2}, \ldots, w_{n}\right)^{T}$ is assumed such that $w_{i}>0$ and $\sum_{i=1}^{n} w_{i}=1$. For $p, q \geq 0$, the weighted geometric HM (WGHM) operator is

$$
\mathrm{WGHM}^{p, q}\left(c_{1}, c_{2}, \ldots, c_{n}\right)=\frac{1}{p+q} \prod_{i=1}^{n} \prod_{j=1}^{n}\left(\left(\mathrm{pc}_{\mathrm{i}}\right)^{w_{i}}+\left(\mathrm{qc}_{j}\right)^{w_{j}}\right)^{2 / n(n+1)}
$$

Although it looks counter-intuitive, the idempotency and reducibility properties are not satisfied by the GWHM and WGHM operators $[26,29]$. To address these limitations, alternative computational procedures could be studied further.

\subsection{Three-Parameter Weighted HM (TPWHM) Operator.}

As mentioned above, the existing HM operators overlook the ternary and higher-order correlations among aggregated arguments. Also, idempotency and reducibility are not satisfied by these operators. Real-world decision-making scenarios would be negatively affected by these drawbacks. To address these drawbacks, we further develop two novel HM operators and investigate four of their key properties. These operators are the three-parameter weighted HM (TPWHM).

Definition 18. Let $\left\{c_{1}, c_{2}, \ldots, c_{n}\right\}$ be a set of $n$ nonnegative numbers. The weight vector is set to be $w=\left(w_{1}, w_{2}, \ldots, w_{n}\right)^{T}$ such that $w_{i}>0$ and $\sum_{i=1}^{n} w_{i}=1$. For $p, q, r \geq 0$, the three-parameter weighted HM (TPWHM) operator is

$$
\operatorname{TPWHM}^{p, q, r}\left(c_{1}, c_{2}, \ldots, c_{n}\right)=\left(\frac{\sum_{i=1}^{n} \sum_{j=i}^{n} \sum_{k=j}^{n} w_{i} w_{j} w_{k} c_{i}^{p} c_{j}^{q} c_{k}^{r}}{\sum_{i=1}^{n} \sum_{j=i}^{n} \sum_{k=j}^{n} w_{i} w_{j} w_{k}}\right)^{1 / p+q+r}
$$

For the TPWHM operator, we show that the reducibility, idempotency, monotonicity, and boundedness properties hold.

Theorem 1 (TPWHM is reducible). The weight vector is set to be $w=(1 / n, 1 / n, \ldots, 1 / n)^{T}$. Hence, the TPWHM is reducible to revised HM (RHM) expressed by

$$
\operatorname{RHM}^{p, q, r}\left(c_{1}, c_{2}, \ldots, c_{n}\right)=\left(\frac{2}{\sum_{\delta=1}^{n} \delta(\delta+1)} \sum_{i=1}^{n} \sum_{j=i}^{n} \sum_{k=j}^{n} c_{i}^{p} c_{j}^{q} c_{k}^{r}\right)^{1 / p+q+r} .
$$

Proof. Based on Definition 18 and given that $w=(1 / n, 1 / n, \ldots, 1 / n)^{T}$, we get 


$$
\begin{aligned}
\operatorname{TPWHM}^{p, q, r}\left(c_{1}, c_{2}, \ldots, c_{n}\right) & =\left(\frac{\sum_{i=1}^{n} \sum_{j=i}^{n} \sum_{k=j}(1 / n)^{3}}{\sum_{i=1}^{n} \sum_{j=i}^{n} \sum_{k=j}^{n}(1 / n)^{3}}\right)^{1 / p+q+r} \\
& =\left(\frac{2}{\sum_{\delta=1}^{n} \delta(\delta+1)} \sum_{i=1}^{n} \sum_{j=i}^{n} \sum_{k=j}^{n} c_{i}^{p} c_{j}^{q} c_{k}^{r}\right)^{1 / p+q+r} \\
& =\operatorname{RHM}^{p, q, r}\left(c_{1}, c_{2}, \ldots, c_{n}\right) .
\end{aligned}
$$

Theorem 2 (TPWHM is idempotent). Given the set of nonnegative numbers $\left\{c_{1}, c_{2}, \ldots, c_{n}\right\}$,

$$
\operatorname{TPWHM}^{p, q, r}\left\{c_{1}, c_{2}, \ldots, c_{n}\right\}=c
$$

could be obtained.

Proof. Given $c=\left\{c_{1}, c_{2}, \ldots, c_{n}\right\}$ and based on equation (13), we get

$$
\begin{aligned}
\operatorname{TPWHM}^{p, q, r}\left\{c_{1}, c_{2}, \ldots, c_{n}\right\} & =\left(\frac{\sum_{i=1}^{n} \sum_{\mathrm{j}=\mathrm{i}}^{n} \sum_{\mathrm{k}=\mathrm{j}}^{n} w_{i} w_{j} w_{k} c^{p} c^{q} c^{r}}{\sum_{i=1}^{n} \sum_{\mathrm{j}=\mathrm{i}}^{n} \sum_{\mathrm{k}=\mathrm{j}}^{n} w_{i} w_{j} w_{k}}\right)^{1 / \mathrm{p}+\mathrm{q}+\mathrm{r}} \\
& =\frac{c\left(\sum_{i=1}^{n} \sum_{\mathrm{j}=\mathrm{i}}^{n} \sum_{\mathrm{k}=\mathrm{j}}^{n} w_{i} w_{j} w_{k}\right)^{1 / \mathrm{p}+\mathrm{q}+\mathrm{r}}}{\left(\sum_{i=1}^{n} \sum_{\mathrm{j}=\mathrm{i}}^{n} \sum_{\mathrm{k}=\mathrm{j}}^{n} w_{i} w_{j} w_{k}\right)^{1 / \mathrm{p}+\mathrm{q}+\mathrm{r}}}=c
\end{aligned}
$$

Theorem 3 (TPWHM is monotone). Two sets of nonnegative numbers are denoted by $c=\left\{c_{1}, c_{2}, \ldots, c\right\}$ and $b=\left\{b_{1}, b_{2}, \ldots, b_{n}\right\}$, respectively. If $c_{i} \geq b_{i}$ for all $i$, then $\operatorname{TPWHM}^{p, q, r}\left\{c_{1}, c_{2}, \ldots, c_{n}\right\} \geq \operatorname{TPWHM}^{p, q, r}\left(b_{1}, b_{2}, \ldots, b_{n}\right)$.
Proof. As $p, q, r \geq 0$ and $c_{i} \geq b_{i}$ for all $i$, then according to equation (13), we have

$$
w_{i} w_{j} w_{k} c_{i}^{p} c_{j}^{q} c_{k}^{r} \geq w_{i} w_{j} w_{k} b_{i}^{p} b_{j}^{q} b_{k}^{r}
$$

Furthermore,

$$
\left(\frac{\sum_{i=1}^{n} \sum_{\mathrm{j}=\mathrm{i}}^{n} \sum_{\mathrm{k}=\mathrm{j}}^{n} w_{i} w_{j} w_{k} c_{i}^{p} c_{j}^{q} c_{k}^{r}}{\sum_{i=1}^{n} \sum_{\mathrm{j}=\mathrm{i}}^{n} \sum_{\mathrm{k}=\mathrm{j}}^{n} w_{i} w_{j} w_{k}}\right)^{1 / \mathrm{p}+\mathrm{q}+\mathrm{r}} \geq\left(\frac{\sum_{i=1}^{n} \sum_{\mathrm{j}=\mathrm{i}}^{n} \sum_{\mathrm{k}=\mathrm{j}}^{n} w_{i} w_{j} w_{k} b_{i}^{p} b_{j}^{q} b_{k}^{r}}{\sum_{i=1}^{n} \sum_{\mathrm{j}=\mathrm{i}}^{n} \sum_{\mathrm{k}=\mathrm{j}}^{n} w_{i} w_{j} w_{k}}\right)^{1 / \mathrm{p}+\mathrm{q}+\mathrm{r}}
$$

Therefore,

$\operatorname{TPWHM}^{p, q, r}\left(c_{1}, c_{2}, \ldots, c_{n}\right) \geq \operatorname{TPWHM}^{p, q, r}\left(b_{1}, b_{2}, \ldots, b_{n}\right)$.

Theorem 4 (TPWHM is bounded). The TPWHM operator output is bounded between highest and lowest scores, i.e.,

$$
\begin{aligned}
\min \left(c_{1}, c_{2}, \ldots, c_{n}\right) & \leq \operatorname{TPWHM}^{p, q, r}\left(c_{1}, c_{2}, \ldots, c_{n}\right) \\
& \leq \max \left(c_{1}, c_{2}, \ldots, c_{n}\right) .
\end{aligned}
$$

Proof. Let $\alpha=\min \left(c_{1}, c_{2}, \ldots, c_{n}\right), \beta=\max \left(c_{1}, c_{2}, \ldots, c_{n}\right)$, and utilizing the idempotent property of TPWHM (Theorem 2), $\operatorname{TPWHM}^{p, q, r}(\alpha, \alpha, \ldots, \alpha)=\alpha, \quad$ TPWHM $p, q, r(\beta, \beta, \ldots, \beta)=\beta$ are obtained.

Since $\alpha \leq c_{i} \leq \beta$ and based on the TPWHM monotonicity (Theorem 3), we have

$$
\begin{aligned}
\operatorname{TPWHM}^{p, q, r}(\alpha, \alpha, \ldots, \alpha) & \leq \operatorname{TPWHM}^{p, q, r}\left(c_{1}, c_{2}, \ldots, c_{n}\right) \leq \operatorname{TPWHM}^{p, q, r}(\beta, \beta, \ldots, \beta) \\
& \leq \operatorname{TPWHM}^{p, q, r}(\beta, \beta, \ldots, \beta) .
\end{aligned}
$$


Therefore, $\alpha \leq \operatorname{TPWHM}^{p, q, r}\left(c_{1}, c_{2}, \ldots, c_{n}\right) \leq \beta$ :

$$
\begin{aligned}
\min \left(c_{1}, c_{2}, \ldots, c_{n}\right) & \leq \operatorname{TPWHM}^{p, q, r}\left(c_{1}, c_{2}, \ldots, c_{n}\right) \\
& \leq \max \left(c_{1}, c_{2}, \ldots, c_{n}\right) .
\end{aligned}
$$

Two special TPWHM cases are defined as follows for different settings of the parameters $p, q$, and $r$.

(1) When $r=0$, we have

$$
\operatorname{TPWHM}^{1,1,0}\left(c_{1}, c_{2}, \ldots, c_{n}\right)=\left(\frac{\sum_{i=1}^{n} \sum_{j=\mathrm{i}}^{n}(n+1-j) w_{i} w_{j} c_{i}^{p} c_{j}^{q}}{\sum_{i=1}^{n} \sum_{j=1}^{n}(n+1-j) w_{i} w_{j}}\right)^{1 / \mathrm{p}+\mathrm{q}}
$$

(2) At a value of 1 for each of $p, q$, and $r$, we have

$$
\operatorname{TPWHM}^{1,1,1}\left(c_{1}, c_{2}, \ldots, c_{n}\right)=\left(\frac{\sum_{i=1}^{n} \sum_{j=i}^{n} \sum_{k=j}^{n} w_{i} w_{j} w_{k} c_{i} c_{j} c_{k}}{\sum_{i=1}^{n} \sum_{j=i}^{n} \sum_{k=j}^{n} w_{i} w_{j} w_{k}}\right)^{1 / 3}
$$

3.3. Three-Parameter Weighted Geometric HM (TPWGHM) Operator. We introduce here the definition, properties, and special cases of TPWGHM.
Definition 19. A set of $n$ nonnegative numbers is denoted by $\left\{c_{1}, c_{2}, \ldots, c_{n}\right\} . w=\left(w_{1}, w_{2}, \ldots, w_{n}\right)^{T}$ such that $w_{i}>0$ and $\sum_{i=1}^{n} w_{i}=1$. For $p, q, r \geq 0$, the three-parameter weighted geometric HM (TPWGHM) operator is

$$
\operatorname{TPWGHM}^{p, q, r}\left(c_{1}, c_{2}, \ldots, c_{n}\right)=\frac{1}{p+q+r} \prod_{i=1}^{n} \prod_{j=i}^{n} \prod_{k=j}^{n}\left(\mathrm{pc}_{i}+\mathrm{qc}_{j}+\mathrm{rc}_{k}\right)^{\frac{2(n+1-j) w_{k}}{\sum_{\delta=1}^{n} \delta(\delta+1) \sum_{b=j}^{n} w_{b}}}
$$

The TPWGHM operator is reducible, idempotent, monotone, and bounded as we show next.
Theorem 5 (TPWGHM is reducible). For $w=(1 / n, 1 / n, \ldots, 1 / n)^{T}$, the TPWGHM can be downgraded to revised geometric HM (RGHM):

$$
\operatorname{RGHM}^{p, q, r}\left(c_{1}, c_{2}, \ldots, c_{n}\right)=\frac{1}{p+q+r} \prod_{i=1}^{n} \prod_{j=i}^{n} \prod_{k=j}^{n}\left(\mathrm{pc}_{i}+\mathrm{qc}_{j}+\mathrm{rc}_{k}\right)^{\frac{2}{\sum_{\delta=1}^{n} \delta(\delta+1)}}
$$

Proof. For $w=(1 / n, 1 / n, \ldots, 1 / n)^{T}$, equation (26) leads to

$$
\begin{aligned}
& \operatorname{TPWGHM}^{p, q, r}\left(c_{1}, c_{2}, \ldots, c_{n}\right) \\
& =\frac{1}{\mathrm{p}+\mathrm{q}+\mathrm{r}} \prod_{i=1}^{n} \prod_{j=i}^{n} \prod_{\mathrm{k}=j}^{n}\left(\mathrm{pc}_{i}+\mathrm{qc}_{j}+\mathrm{rc}_{k}\right) \frac{2(n+1-j) 1 / n}{\sum_{\delta=1}^{n} \delta(\delta+1)(n+1-j) 1 / n} \\
& =\frac{1}{\mathrm{p}+\mathrm{q}+\mathrm{r}} \prod_{i=1}^{n} \prod_{j=i}^{n} \prod_{\mathrm{k}=\mathrm{j}}^{n}\left(\mathrm{pc}_{i}+\mathrm{qc}_{j}+\mathrm{rc}_{k}\right) \frac{2}{\sum_{\delta=1}^{n} \delta(\delta+1)} \\
& =\operatorname{RGHM}^{p, q, r}\left(c_{1}, c_{2}, \ldots, c_{n}\right) .
\end{aligned}
$$


Therefore, the TPWGHM operator is reducible.

Theorem 6 (TPWGHM is idempotent). For the nonnegative number set $\left(c_{1}, c_{2}, \ldots, c_{n}\right)$, the TPWGHM is idempotent, i.e.,

$$
\operatorname{TPWGHM}^{p, q, r}\left(c_{1}, c_{2}, \ldots, c_{n}\right)=c \text {. }
$$

Proof. For the nonnegative numbers $\left(c_{1}, c_{2}, \ldots, c_{n}\right)$, equation (26) leads to

$$
\begin{aligned}
& \text { TPWGHM } M^{p, q, r}\left(c_{1}, c_{2}, \ldots, c_{n}\right) \\
& =\frac{1}{\mathrm{p}+\mathrm{q}+\mathrm{r}} \prod_{i=1}^{n} \prod_{j=i}^{n} \prod_{\mathrm{k}=\mathrm{j}}^{n}(\mathrm{pc}+\mathrm{qc}+\mathrm{rc}) \\
& =\frac{1}{\mathrm{p}+\mathrm{q}+\mathrm{r}}(\mathrm{pc}+\mathrm{qc}+\mathrm{rc}) \frac{2(n+1-j) w_{k}}{\sum_{\delta=1}^{n} \delta(\delta+1) \sum_{b=j}^{n} w_{b}} \\
& =\frac{1}{\mathrm{p}+\mathrm{q}+\mathrm{r}}(\mathrm{pc}+\mathrm{qc}+\mathrm{rc}) \\
& =\frac{1}{\mathrm{p}+\mathrm{q}+\mathrm{r}}(\mathrm{pc}+\mathrm{qc}+\mathrm{rc}) \sum_{i=1}^{n} \sum_{j=i}^{n} \sum_{k=j}^{n} \frac{(n+1-j) w_{k}}{\sum_{b=j}^{n} w_{b}} \\
& =\frac{1}{\mathrm{p}+\mathrm{q}+\mathrm{r}}(\mathrm{pc}+\mathrm{qc}+\mathrm{rc})=c . \\
& \sum_{i=1}^{n} \sum_{j=i}^{n} \frac{(n+1-j) \sum_{k=j}^{n} w_{k}}{\sum_{b=j}^{n} w_{b}}
\end{aligned}
$$

Theorem 7 (TPWGHM is monotone). For any two sets of nonnegative numbers $\left\{c_{1}, c_{2}, \ldots, c_{n}\right\}$ and $\left\{b_{1}, b_{2}, \ldots, b_{n}\right\}$, if $c_{i} \geq b_{i}$ for all $i$,

$\operatorname{TPWGHM}^{p, q, r}\left(c_{1}, c_{2}, \ldots, c_{n}\right) \geq \operatorname{TPWGHM}^{p, q, r}\left(b_{1}, b_{2}, \ldots, b_{n}\right)$

are obtained.

Proof. As $p, q, r \geq 0$ and $c_{i} \geq b_{i}$ for all $i$, equation (26) leads to

$$
\left(\mathrm{pc}_{i}+\mathrm{qc}_{j}+\mathrm{rc}_{k}\right)^{\frac{2(n+1-j) w_{k}}{\sum_{\delta=1}^{n} \delta(\delta+1) \sum_{b=j}^{n} w_{b}}} \geq\left(\mathrm{pb}_{i}+\mathrm{qb}_{j}+\mathrm{rb}_{k}\right)^{\frac{2(n+1-j) w_{k}}{\sum_{\delta=1}^{n} \delta(\delta+1) \sum_{b=j}^{n} w_{b}}}
$$

Furthermore,

$$
\frac{1}{p+q+r} \prod_{i=1}^{n} \prod_{j=i}^{n} \prod_{k=j}^{n}\left(\mathrm{pc}_{i}+\mathrm{qc}_{j}+\mathrm{rc}_{k}\right)^{\frac{2(n+1-j) w_{k}}{\sum_{\delta=1}^{n} \delta(\delta+1) \sum_{b=j}^{n} w_{b}}} \geq \frac{1}{p+q+r} \prod_{i=1}^{n} \prod_{j=i}^{n} \prod_{k=j}^{n}\left(\mathrm{pb}_{i}+\mathrm{qb}_{j}+\mathrm{rb}_{k}\right)^{\frac{2(n+1-j) w_{k}}{\sum_{\delta=1}^{n} \delta(\delta+1) \sum_{b=j}^{n} w_{b}}}
$$


Therefore,

$\operatorname{TPWGHM}^{p, q, r}\left(c_{1}, c_{2}, \ldots, c_{n}\right) \geq \operatorname{TPWGHM}^{p, q, r}\left(b_{1}, b_{2}, \ldots, b_{n}\right)$.

Theorem 8 (TPWGHM is bounded). The TPWGHM operator output is bounded between maximum and minimum values, i.e.,

$$
\begin{aligned}
\min \left(c_{1}, c_{2}, \ldots, c_{n}\right) & \leq \operatorname{TPWGHM}^{p, q, r}\left(c_{1}, c_{2}, \ldots, c_{n}\right) \\
& \leq \max \left(c_{1}, c_{2}, \ldots, c_{n}\right) .
\end{aligned}
$$

Proof. Let $\alpha=\min \left(c_{1}, c_{2}, \ldots, c_{n}\right)$ and $\beta=\max \left(c_{1}, c_{2}, \ldots\right.$, $c_{n}$ ). The TPWGHM idempotency (Theorem 6) leads to

$\operatorname{TPWGHM}^{p, q, r}(\alpha, \alpha, \ldots, \alpha)=\alpha, \operatorname{TPWGHM}^{p, q, r}(\beta, \ldots, \beta)=\beta$.
Since $\alpha \leq c_{i} \leq \beta$, the TPWGHM monotonicity (Theorem 7) leads to

$$
\begin{aligned}
\operatorname{TPWGHM}^{p, q, r}(\alpha, \alpha, \ldots, \alpha) & \leq \operatorname{TPWGHM}^{p, q, r}\left(c_{1}, c_{2}, \ldots, c_{n}\right) \\
& \leq \operatorname{TPWGHM}^{p, q, r}(\beta, \beta, \ldots \beta) .
\end{aligned}
$$

This is equivalent to $\alpha \leq \operatorname{TPWGHM} M^{p, q, r}\left(c_{1}, c_{2}, \cdots\right.$, $\left.c_{n}\right) \leq \beta$. Then, we get

$$
\begin{aligned}
\min \left(c_{1}, c_{2}, \ldots, c_{n}\right) & \leq \operatorname{TPWGHM}^{p, q, r}\left(c_{1}, c_{2}, \ldots, c_{n}\right) \\
& \leq \max \left(c_{1}, c_{2}, \ldots, c_{n}\right) .
\end{aligned}
$$

Based on parameters $p, q$ and $r$, two special TPWGHM cases follow.

(1) When $r=0$, we have

$$
\operatorname{TPWGHM}^{p, q, 0}\left(c_{1}, c_{2}, \ldots, c_{n}\right)=\frac{1}{p+q} \prod_{i=1}^{n} \prod_{j=i}^{n}\left(\mathrm{pc}_{i}+\mathrm{qc}_{j}\right)^{\frac{2(n+1-j)}{\sum_{\delta=1}^{n} \delta(\delta+1)}}
$$

(2) At a value of 1 for each of $p, q$, and $r$, we have

$$
\operatorname{TPWGHM}^{1,1,1}\left(c_{1}, c_{2}, \ldots, c_{n}\right)=\left(\frac{\sum_{i=1}^{n} \sum_{\mathrm{j}=\mathrm{i}}^{n} \sum_{\mathrm{k}=\mathrm{j}}^{n} w_{i} w_{j} w_{k}, c_{i}, c_{j}, c_{k}}{\sum_{i=1}^{n} \sum_{\mathrm{j}=\mathrm{i}}^{n} \sum_{\mathrm{k}=\mathrm{j}}^{n} w_{i} w_{j} w_{k}}\right)^{1 / 3}
$$

\section{Three-Parameter Heronian Mean Operators for IVHFL Information}

While crisp values can be aggregated with the TPWHM and TPWGHM, these operators fail to combine IVHFL information. Here, the limitation could be addressed through the extension of these two operators to novel HM operators, namely, IVHFLTPWHM and IVHFLTPWHM.
4.1. Interval-Valued Hesitant Fuzzy Linguistic TPWHM (IVHFLTPWHM) Operator

Definition 20. A set of IVHFLEs is defined by $\left\{c_{1}, c_{2}, \ldots, c_{n}\right\}$. The weight is set to be $w=\left(w_{1}, w_{2}, \ldots, w_{n}\right)^{T}$ such that $w_{i}>0$ and $\sum_{i=1}^{n} w_{i}=1$. For $p, q, r \geq 0$, the operator of (IVHFLTPWHM) is defined as

$$
\operatorname{IVHFLTPWHM}^{p, q, r}\left(c_{1}, c_{2}, \ldots, c_{n}\right)=\left(\frac{1}{\sum_{i=1}^{n} \sum_{j=i}^{n} \sum_{k=j}^{n} w_{i} w_{j} w_{k}} \stackrel{\oplus}{i=1}_{j=i}^{n} \oplus_{j=j}^{n} \mathfrak{\oplus}_{i}^{n} w_{j} w_{k}\left(c_{i}^{p} \otimes c_{j}^{q} \otimes c_{k}^{r}\right)\right)^{1 / p+q+r}
$$


Theorem 9. A set of IVHFLEs is defined by $c_{i}=\left\langle s_{\theta\left(c_{i}\right)}, \Gamma_{\theta\left(c_{i}\right)}\right\rangle(i=1,2, \ldots, n)$. A weight vector is set to be $w=\left(w_{1}, w_{2}, \ldots, w_{n}\right)^{T}$ satisfying $w_{i}>0$ and $\sum_{i=1}^{n} w_{i}=1$.
Utilizing IVHFLTPWHM, the combined outcome is an IVHFLE. For $p, q, r \geq 0$,

$$
\begin{aligned}
& \operatorname{IVHFLTPWHM}^{p, q, r}\left(c_{1}, c_{2}, \ldots, c_{n}\right)=\left\langle s\left(\sum_{i=1}^{n} \sum_{j=i}^{n} \sum_{k=j}^{n} w_{i} w_{j} w_{k} \theta\left(c_{i}\right)^{p} \theta\left(c_{j}\right)^{q} \theta\left(c_{k}\right)^{r}\right)^{1 / p+q+r}, \cup_{\gamma_{\theta\left(a_{i}\right)} \in \Gamma_{\theta\left(c_{i}\right)}, \gamma_{\theta}\left(c_{j}\right)}^{\in \Gamma_{\theta\left(c_{j}\right)}, \gamma_{\theta\left(c_{k}\right)} \in \Gamma_{\theta\left(c_{k}\right)}},\right. \\
& {\left[\left(1-\left(1-\left(1-\prod_{i=1}^{n} \prod_{j=i}^{n} \prod_{k=j}^{n}\left(1-\left(\gamma_{\theta\left(c_{i}\right)}^{L}\right)^{p}\left(\gamma_{\theta\left(c_{j}\right)}^{L}\right)^{q}\left(\gamma_{\theta\left(c_{k}\right)}^{L}\right)^{r}\right)^{w_{i} w_{j} w_{k}}\right)\right)^{\frac{1}{\sum_{i=1}^{n} \sum_{j=i}^{n} \sum_{k=j}^{n} w_{i} w_{j} w_{k}}}\right],\right.} \\
& \left.\left(1-\left(1-\left(1-\prod_{i=1}^{n} \prod_{j=i}^{n} \prod_{k=j}^{n}\left(1-\left(\gamma_{\theta\left(c_{i}\right)}^{U}\right)^{p}\left(\gamma_{\theta\left(c_{j}\right)}^{U}\right)^{q}\left(\gamma_{\theta\left(c_{k}\right)}^{U}\right)^{r}\right)^{w_{i} w_{j} w_{k}}\right)\right)^{\frac{1}{\sum_{i=1}^{n} \sum_{j=i}^{n} \sum_{k=j}^{n} w_{i} w_{j} w_{k}}}\right]\right\rangle \\
& \operatorname{IVHFLTPWHM}^{p, q, r}\left(c_{1}, c_{2}, \ldots, c_{n}\right)=c .
\end{aligned}
$$
tent, monotone, and bounded.

Theorem 10 (IVHFLTPWHM is idempotent). Set the Proof. As $a=\left\{c_{1}, c_{2}, \ldots, c_{n}\right\}$, equation (41) leads to nonnegative numbers $c=\left\{c_{1}, c_{2}, \ldots, c_{n}\right\}$ such that $c=\left\langle s_{\theta(c)}, \Gamma_{\theta(c)}\right\rangle$. Then, we have

$$
\begin{aligned}
\operatorname{IVHFLTPWHM}^{p, q, r}\left(c_{1}, c_{2}, \ldots, c_{n}\right) & =\left(\frac{1}{\sum_{i=1}^{n} \sum_{\mathrm{j}=\mathrm{i}}^{n} \sum_{\mathrm{k}=\mathrm{j}}^{n} w_{i} w_{j} w_{k}} \underset{i=1}{\oplus} \underset{j=i}{\oplus} \underset{\mathrm{k}=\mathrm{j}}{\oplus} w_{i} w_{j} w_{k}\left(c^{p+q+r}\right)\right)^{1 / p+q+r} \\
& =\frac{c\left(\sum_{i=1}^{n} \sum_{j=i}^{n} \sum_{k=j}^{n} w_{i} w_{j} w_{k}\right)^{1 / p+q+r}}{\left(\sum_{i=1}^{n} \sum_{j=i}^{n} \sum_{k=j}^{n} w_{i} w_{j} w_{k}\right)^{1 / p+q+r}}=c .
\end{aligned}
$$

Theorem 11 (IVHFLTPWHM is monotone). Let $\gamma_{\theta\left(c_{i}\right)}=\left[\gamma_{\theta\left(c_{i}\right)}^{L}, \gamma_{\theta\left(c_{i}\right)}^{U}\right] \in \Gamma_{\theta\left(c_{i}\right)}$ and $\gamma_{\theta\left(b_{i}\right)}=\left[\gamma_{\theta\left(b_{i}\right)}^{L}, \gamma_{\theta\left(b_{i}\right)}^{U}\right] \in$ $c_{i}=\left\langle s_{\theta\left(c_{i}\right)}, \Gamma_{\theta\left(c_{i}\right)}\right\rangle$, and $b_{i}=\left\langle s_{\theta\left(b_{i}\right)}, \Gamma_{\theta\left(b_{i}\right)}\right\rangle$ be two IVHFLEs, $\Gamma_{\theta\left(b_{i}\right)}$. If $c_{i} \geq b_{i}$ for all $i$, then

$$
\operatorname{IVHFLTPWHM}^{p, q, r}\left(c_{1}, c_{2}, \ldots, c_{n}\right) \geq \operatorname{IVHFLTPWHM}^{p, q, r}\left(b_{1}, b_{2}, \ldots, b_{n}\right)
$$

Proof. As $\gamma_{\theta\left(c_{i}\right)}^{L} \geq \gamma_{\theta\left(b_{i}\right)}^{L}$ and $\gamma_{\theta\left(c_{i}\right)}^{U} \geq \gamma_{\theta\left(b_{i}\right)}^{U}$ for all $i$ and $p, q, r \geq 0$, according to equation (42), we have 


$$
\begin{gathered}
\left(\gamma_{\theta\left(c_{i}\right)}^{L}\right)^{p}\left(\gamma_{\theta\left(c_{j}\right)}^{L}\right)^{q}\left(\gamma_{\theta\left(c_{k}\right)}^{L}\right)^{r} \geq\left(\gamma_{\theta\left(b_{i}\right)}^{L}\right)^{p}\left(\gamma_{\theta\left(b_{j}\right)}^{L}\right)^{q}\left(\gamma_{\theta\left(b_{k}\right)}^{L}\right)^{r}, \\
\left(1-\left(\gamma_{\theta\left(c_{i}\right)}^{L}\right)^{p}\left(\gamma_{\theta\left(c_{j}\right)}^{L}\right)^{q}\left(\gamma_{\theta\left(c_{k}\right)}^{L}\right)^{r}\right)^{w_{i} w_{j} w_{k}} \leq\left(1-\left(\gamma_{\theta\left(b_{i}\right)}^{L}\right)^{p}\left(\gamma_{\theta\left(b_{j}\right)}^{L}\right)^{q}\left(\gamma_{\theta\left(b_{k}\right)}^{L}\right)^{r}\right)^{w_{i} w_{j} w_{k}} .
\end{gathered}
$$

Furthermore,

$$
\begin{aligned}
& \left(1-\left(1-\left(1-\prod_{i=1}^{n} \prod_{j=i}^{n} \prod_{k=j}^{n}\left(1-\left(\gamma_{\theta\left(a_{i}\right)}^{L}\right)^{p}\left(\gamma_{\theta\left(a_{j}\right)}^{L}\right)^{q}\left(\gamma_{\theta\left(a_{k}\right)}^{L}\right)^{r}\right)^{w_{i} w_{j} w_{k}}\right)\right)^{\left.\frac{1}{\sum_{i=1}^{n} \sum_{j=i}^{n} \sum_{k=j}^{n} w_{i} w_{j} w_{k}}\right)^{1 /(p+q+r)}}\right. \\
& \geq\left(1-\left(1-\left(1-\prod_{i=1}^{n} \prod_{j=i}^{n} \prod_{k=j}^{n}\left(1-\left(\gamma_{\theta\left(b_{i}\right)}^{L}\right)^{p}\left(\gamma_{\theta\left(b_{j}\right)}^{L}\right)^{q}\left(\gamma_{\theta\left(b_{k}\right)}^{L}\right)^{r}\right)^{w_{i} w_{j} w_{k}}\right)\right)^{\left.\frac{1}{\sum_{i=1}^{n} \sum_{j=i}^{n} \sum_{k=j}^{n} w_{i} w_{j} w_{k}}\right)^{1 /(p+q+r)}} .\right.
\end{aligned}
$$

Similarly, the inequality is expressed as

$$
\begin{aligned}
& \left(1-\left(1-\left(1-\prod_{i=1}^{n} \prod_{j=i}^{n} \prod_{k=j}^{n}\left(1-\left(\gamma_{\theta\left(a_{i}\right)}^{U}\right)^{p}\left(\gamma_{\theta\left(a_{j}\right)}^{U}\right)^{q}\left(\gamma_{\theta\left(a_{k}\right)}^{U}\right)^{r}\right)^{w_{i} w_{j} w_{k}}\right)\right)^{\left.\frac{1}{\sum_{i=1}^{n} \sum_{j=i}^{n} \sum_{k=j}^{n} w_{i} w_{j} w_{k}}\right)^{1 / p+q+r}}\right. \\
& \geq\left(1-\left(1-\left(1-\prod_{i=1}^{n} \prod_{j=i}^{n} \prod_{k=j}^{n}\left(1-\left(\gamma_{\theta\left(b_{i}\right)}^{U}\right)^{p}\left(\gamma_{\theta\left(b_{j}\right)}^{U}\right)^{q}\left(\gamma_{\theta\left(b_{k}\right)}^{U}\right)^{r}\right)^{w_{i} w_{j} w_{k}}\right)\right)^{\left.\frac{1}{\sum_{i=1}^{n} \sum_{j=i}^{n} \sum_{k=j}^{n} w_{i} w_{j} w_{k}}\right)^{1 / p+q+r}} .\right.
\end{aligned}
$$

In addition, the summation is expressed by

$$
\mathcal{S}\left(\sum_{i=1}^{n} \sum_{j=i}^{n} \sum_{k=j}^{n} w_{i} w_{j} w_{k} \theta\left(c_{i}\right)^{p} \theta\left(c_{j}\right)^{q} \theta\left(c_{k}\right)^{r}\right)^{1 / p+q+r} \geq \mathcal{S}\left(\sum_{i=1}^{n} \sum_{j=i}^{n} \sum_{k=j}^{n} w_{i} w_{j} w_{k} \theta\left(b_{i}\right)^{p} \theta\left(b_{j}\right)^{q} \theta\left(b_{k}\right)^{r}\right)^{1 / p+q+r}
$$

So, operators are expressed by

$$
\operatorname{IVHFLTPWHM}^{p, q, r}\left(c_{1}, c_{2}, \ldots, c_{n}\right) \geq \operatorname{IVHFLTPWHM}^{p, q, r}\left(b_{1}, b_{2}, \ldots, b_{n}\right) .
$$

Theorem 12 (IVHFLTPWHM is bounded). The IVHFLTPWHM operator output is bounded between highest and lowest scores, i.e.,

$$
\min \left(c_{1}, c_{2}, \ldots, c_{n}\right) \leq \operatorname{IVHFLTPWHM}^{p, q, r}\left(c_{1}, c_{2}, \ldots, c_{n}\right) \leq \max \left(c_{1}, c_{2}, \ldots, c_{n}\right)
$$


Proof. Let $\alpha=\min \left(c_{1}, c_{2}, \ldots, c_{n}\right), \beta=\max \left(c_{1}, c_{2}, \ldots, c_{n}\right)$. According to Theorem 10, IVHFLTPWHM ${ }^{p, q, r}$ $(\alpha, \alpha, \ldots, \alpha)=\alpha$, and $\operatorname{IVHFLTPWHM}^{p, q, r}(\beta, \beta, \ldots, \beta)=\beta$.
Since $\alpha \leq c_{i} \leq \beta$ and based on Theorem 11, we have

$$
\begin{aligned}
\operatorname{IVHFLTPWHM}^{p, q, r}(\alpha, \alpha, \ldots, \alpha) & \leq \operatorname{IVHFLTPWHM}^{p, q, r}\left(c_{1}, c_{2}, \ldots, c_{n}\right) \\
& \leq \operatorname{IVHFLTPWHM}^{p, q, r}(\beta, \beta, \ldots, \beta)
\end{aligned}
$$

Therefore, $\quad \alpha \leq$ IVHFLTPWHM $^{p, q, r}\left(c_{1}, c_{2}, \ldots, c_{n}\right) \leq \beta$, and thus

$$
\min \left(c_{1}, c_{2}, \ldots, c_{n}\right) \leq \operatorname{IVHFLTPWHM}^{p, q, r}\left(c_{1}, c_{2}, \ldots, c_{n}\right) \leq \max \left(c_{1}, c_{2}, \ldots, c_{n}\right)
$$

4.2. Interval-Valued Hesitant Fuzzy Linguistic TPWGHM (IVHFLTPWGHM) Operator
Definition 21. A set of IVHFLEs is defined by $c=\left\{c_{1}, c_{2}, \ldots, c_{n}\right\}$, and let $w=\left(w_{1}, w_{2}, \ldots, w_{n}\right)^{T}$ denote a weight vector satisfying $w_{i}>0$ and $\sum_{i=1}^{n} w_{i}=1$. For $p, q, r \geq 0$, the operator called IVHFLTPWGHM is

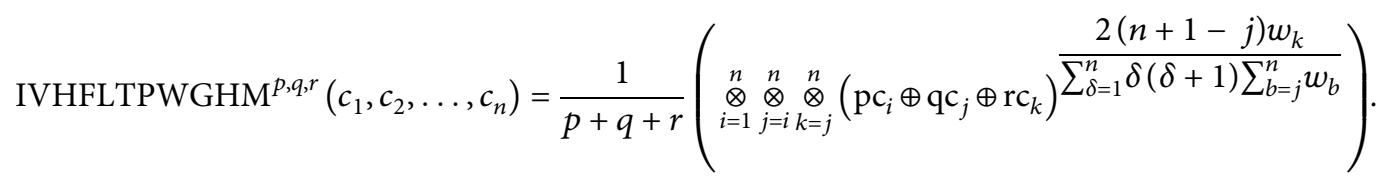

Theorem 13. Let $p, q, r \geq 0$ and $c_{i}=\left\langle s_{\theta\left(c_{i}\right)}, \Gamma_{\theta\left(c_{i}\right)}\right\rangle$ $(i=1,2, \ldots, n)$ denote $a$ set of IVHFLES with $w=\left(w_{1}, w_{2}, \ldots, w_{n}\right)^{T}$ satisfying $w_{i}>0$ and $\sum_{i=1}^{n} w_{i}=1$. For the IVHFLTPWGHM, the total is also an IVHFLE. Then,

$$
\begin{aligned}
& \operatorname{IVHFLTPWGHM}^{p, q, r}\left(c_{1}, c_{2}, \ldots, c_{n}\right) \\
& \begin{aligned}
= & \left\langle s\left(\frac{\prod_{i=1}^{n} \prod_{\mathrm{j}=\mathrm{i}}^{n} \prod_{\mathrm{k}=\mathrm{j}}^{n}\left(p \theta\left(c_{i}\right)+q \theta\left(c_{j}\right)+r \theta\left(c_{k}\right)\right)\left(2(n+1-j) w_{k} / \sum_{\delta=1}^{n} \delta(\delta+1) \sum_{b=j}^{n} w_{b}\right)}{p+q+r}\right), \cup_{\gamma_{\theta\left(c_{i}\right)} \in \Gamma_{\theta\left(c_{i}\right)}, \gamma_{\theta\left(c_{j}\right)} \in \Gamma_{\theta\left(c_{j}\right)}, \gamma_{\theta\left(c_{k}\right)} \in \Gamma_{\theta\left(c_{k}\right)}}\right. \\
& {\left[1-\left(1-\prod_{i=1}^{n} \prod_{\mathrm{j}=\mathrm{i}}^{n} \prod_{\mathrm{k}=\mathrm{j}}^{n}\left(1-\left(1-\gamma_{\theta\left(c_{i}\right)}^{\mathrm{L}}\right)^{p}\left(1-\gamma_{\theta\left(c_{j}\right)}^{\mathrm{L}}\right)^{q}\left(1-\gamma_{\theta\left(c_{k}\right)}^{\mathrm{L}}\right)^{r}\right)^{2(n+1-j) w_{k} / \sum_{\delta=1}^{n} \delta(\delta+1) \sum_{b=j}^{n} w_{b}}\right)^{1 / p+q+r},\right.}
\end{aligned}
\end{aligned}
$$

$$
\left.\left.1-\left(1-\prod_{i=1}^{n} \prod_{\mathrm{j}=\mathrm{i}}^{n} \prod_{\mathrm{k}=\mathrm{j}}^{n}\left(1-\left(1-\gamma_{\theta\left(c_{i}\right)}^{\mathrm{U}}\right)^{p}\left(1-\gamma_{\theta\left(c_{j}\right)}^{\mathrm{U}}\right)^{q}\left(1-\gamma_{\theta\left(c_{k}\right)}^{\mathrm{U}}\right)^{r}\right)^{2(n+1-j) w_{k} / \sum_{\delta=1}^{n} \delta(\delta+1) \sum_{b=j}^{n} w_{b}}\right)^{1 / p+q+r}\right]\right\rangle
$$

Moreover, the IVHFLTPWGHM operator is idempotent, monotone, and bounded as we show next.

Theorem 14 (IVHFLTPWGHM is idempotent). Let $c=\left\{c_{1}, c_{2}, \ldots, c_{n}\right\}$, where $c=\left\langle s_{\theta(c)}, \Gamma_{\theta(c)}\right\rangle$. We have

$$
\operatorname{IVHFLTPWGH} M^{p, q, r}\left(c_{1}, c_{2}, \cdots, c_{n}\right)=c \text {. }
$$




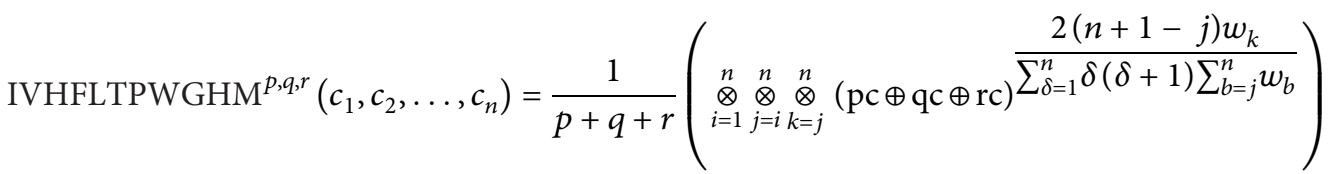

$$
\begin{aligned}
& =\frac{1}{p+q+r}(\mathrm{pc}+\mathrm{qc}+\mathrm{rc}) \frac{2}{\sum_{\delta=1}^{n} \delta(\delta+1)} \sum_{i=1}^{n} \sum_{j=i}^{n} \sum_{k=j}^{n} \frac{(n+1-j) w_{k}}{\sum_{b=j}^{n} w_{b}} \\
& =\frac{1}{p+q+r}(\mathrm{pc}+\mathrm{qc}+\mathrm{rc}) \frac{2}{\sum_{\delta=1}^{n} \delta(\delta+1)} \sum_{i=1}^{n}((n+1-i)+\cdots+1) \\
& =\frac{1}{\mathrm{p}+\mathrm{q}+\mathrm{r}}(\mathrm{pc}+\mathrm{qc}+\mathrm{rc})=c \text {. }
\end{aligned}
$$

Theorem 15 (IVHFLTPWGHM is monotone). Let $\gamma_{\theta\left(c_{i}\right)}=\left[\gamma_{\theta\left(c_{i}\right)}^{L}, \gamma_{\theta\left(c_{i}\right)}^{U}\right] \in \Gamma_{\theta\left(b_{i}\right)}$, and $\gamma_{\theta\left(b_{i}\right)}=\left[\gamma_{\theta\left(b_{i}\right)}^{L}, \gamma_{\theta\left(b_{i}\right)}^{U}\right] \epsilon$ $c_{i}=\left\langle s_{\theta\left(c_{i}\right)}, \Gamma_{\theta\left(c_{i}\right)}\right\rangle$, and $b_{i}=\left\langle s_{\theta\left(b_{i}\right)}, \Gamma_{\theta\left(b_{i}\right)}\right\rangle$ be two IVHFLEs, $\quad \Gamma_{\theta\left(b_{i}\right)}$. If $c_{i} \geq b_{i}$ for all $i$, then

$$
\operatorname{IVHFLTPWHM}^{p, q, r}\left(c_{1}, c_{2}, \ldots, c_{n}\right) \geq \operatorname{IVHFLTPWHM}^{p, q, r}\left(b_{1}, b_{2}, \ldots, b_{n}\right)
$$

Proof. As $\gamma_{\theta\left(c_{i}\right)}^{L} \geq \gamma_{\theta\left(b_{i}\right)}^{L}$ and $\gamma_{\theta\left(c_{i}\right)}^{U} \geq \gamma_{\theta\left(b_{i}\right)}^{U}$ for all $i$ and $p, q, r \geq 0$, according to equation (57), we have

$$
\begin{gathered}
\left(1-\gamma_{\theta\left(c_{i}\right)}^{L}\right)^{p}\left(1-\gamma_{\theta\left(c_{j}\right)}^{L}\right)^{q}\left(1-\gamma_{\theta\left(c_{k}\right)}^{L}\right)^{r} \leq\left(1-\gamma_{\theta\left(b_{i}\right)}^{L}\right)^{p}\left(1-\gamma_{\theta\left(b_{j}\right)}^{L}\right)^{q}\left(1-\gamma_{\theta\left(b_{k}\right)}^{L}\right)^{r} \\
\left.\left(1-\left(1-\gamma_{\theta\left(c_{i}\right)}^{L}\right)\right)^{p}\left(1-\gamma_{\theta\left(c_{j}\right)}^{L}\right)^{q}\left(1-\gamma_{\theta\left(c_{k}\right)}^{L}\right)^{r}\right)^{2(n+1-j) w_{k} / \sum_{\delta=1}^{n} \delta(\delta+1) \sum_{b=j}^{n} w_{b}} \\
\geq\left(1-\left(1-\gamma_{\theta\left(b_{i}\right)}^{L}\right)^{p}\left(1-\gamma_{\theta\left(b_{j}\right)}^{L}\right)^{q}\left(1-\gamma_{\theta\left(b_{k}\right)}^{L}\right)^{r}\right)^{2(n+1-j) w_{k} \sum_{\delta=1}^{n} \delta(\delta+1)} \sum_{b=j}^{n} w_{b}
\end{gathered}
$$

from which we conclude that

$$
\begin{aligned}
& \left(1-\left(1-\left(1-\prod_{i=1}^{n} \prod_{j=i}^{n} \prod_{k=j}^{n}\left(1-\left(\gamma_{\theta\left(c_{i}\right)}^{L}\right)^{p}\left(\gamma_{\theta\left(c_{j}\right)}^{L}\right)^{q}\left(\gamma_{\theta\left(c_{k}\right)}^{L}\right)^{r}\right)^{w_{i} w_{j} w_{k}}\right)\right)^{1 / \sum_{i=1}^{n} \sum_{j=i}^{n} \sum_{k=j}^{n} w_{i} w_{j} w_{k}}\right)^{1 / p+q+r} \\
& \geq\left(1-\left(1-\left(1-\prod_{i=1}^{n} \prod_{j=i}^{n} \prod_{k=j}^{n}\left(1-\left(\gamma_{\theta\left(b_{i}\right)}^{L}\right)^{p}\left(\gamma_{\theta\left(b_{j}\right)}^{L}\right)^{q}\left(\gamma_{\theta\left(b_{k}\right)}^{L}\right)^{r}\right)^{w_{i} w_{j} w_{k}}\right)\right)^{1 / \sum_{i=1}^{n} \sum_{j=i}^{n} \sum_{k=j}^{n} w_{i} w_{j} w_{k}}\right)^{1 / p+q+r} .
\end{aligned}
$$


In the same way,

$$
\begin{aligned}
1 & -\left(1-\prod_{i=1}^{n} \prod_{j=i}^{n} \prod_{k=j}^{n}\left(1-\left(1-\gamma_{\theta\left(c_{i}\right)}^{L}\right)^{p}\left(1-\gamma_{\theta\left(c_{j}\right)}^{L}\right)^{q}\left(1-\gamma_{\theta\left(c_{k}\right)}^{L}\right)^{r}\right)^{2(n+1-j) w_{k} / \sum_{\delta=1}^{n} \delta(\delta+1) \sum_{b=j}^{n} w_{b}}\right)^{1 / p+q+r} \\
& \geq 1-\left(1-\prod_{i=1}^{n} \prod_{j=i}^{n} \prod_{k=j}^{n}\left(1-\left(1-\gamma_{\theta\left(b_{i}\right)}^{L}\right)^{p}\left(1-\gamma_{\theta\left(b_{j}\right)}^{L}\right)^{q}\left(1-\gamma_{\theta\left(b_{k}\right)}^{L}\right)^{r}\right)^{2(n+1-j) w_{k} / \sum_{\delta=1}^{n} \delta(\delta+1) \sum_{b=j}^{n} w_{b}}\right)^{1 / p+q+r}
\end{aligned}
$$

In addition,

$$
\begin{gathered}
\mathcal{S}\left(\frac{\prod_{i=1}^{n} \prod_{j=i}^{n} \prod_{\mathrm{k}=j}^{n}\left(p \theta\left(c_{i}\right)+q \theta\left(c_{j}\right)+r \theta\left(c_{k}\right)\right)\left(2(n+1-j) w_{k} / \sum_{\delta=1}^{n} \delta(\delta+1) \sum_{b=j}^{n} w_{b}\right)}{p+q+r}\right) \\
\quad \geq s\left(\frac{\prod_{i=1}^{n} \prod_{\mathrm{j}=\mathrm{i}}^{n} \prod_{\mathrm{k}=\mathrm{j}}^{n}\left(p \theta\left(b_{i}\right)+q \theta\left(b_{j}\right)+r \theta\left(b_{k}\right)\right)\left(2(n+1-j) w_{k} / \sum_{\delta=1}^{n} \delta(\delta+1) \sum_{b=j}^{n} w_{b}\right)}{p+q+r}\right)
\end{gathered}
$$

So, we have

$$
\begin{aligned}
& \operatorname{IVHFLTPWGHM}^{p, q, r}\left(c_{1}, c_{2}, \ldots, c_{n}\right) \\
& \geq \operatorname{IVHFLTPWGHM}^{p, q, r}\left(b_{1}, b_{2}, \ldots, b_{n}\right) .
\end{aligned}
$$

Theorem 16 (IVHFLTPWGHM is bounded). The IVHFLTPWGHM operator outcome $t$ is contained between highest and lowest scores, i.e.,

$$
\min \left(c_{1}, c_{2}, \ldots, c_{n}\right) \leq \operatorname{IVHFLTPWGHM}^{p, q, r}\left(c_{1}, c_{2}, \ldots, c_{n}\right) \leq \max \left(c_{1}, c_{2}, \ldots, c_{n}\right)
$$

Proof. Let $\alpha=\min \left(c_{1}, c_{2}, \ldots, c_{n}\right), \beta=\max \left(c_{1}, c_{2}, \ldots, c_{n}\right)$.

On the basis of Theorem 14, we have

$$
\operatorname{IVHFLTPWGHM}^{p, q, r}(\alpha, \alpha, \ldots, \alpha)=\alpha, \operatorname{IVHFLTPWGHM}^{p, q, r}(\beta, \beta, \ldots, \beta)=\beta
$$

Since $\alpha \leq c_{i} \leq \beta$ and based on Theorem 15, we have

$$
\begin{aligned}
\operatorname{IVHFLTPWGHM}^{p, q, r}(\alpha, \alpha, \ldots, \alpha) \leq & \operatorname{IVHFLTPWGHM}^{p, q, r}\left(c_{1}, c_{2}, \ldots, c_{n}\right) \\
& \leq \operatorname{IVHFLTPWGHM}^{p, q, r}(\beta, \beta, \ldots, \beta) .
\end{aligned}
$$
and

Therefore, $\alpha \leq \operatorname{IVHFLTPWGHM}^{p, q, r}\left(c_{1}, c_{2}, \ldots, c_{n}\right) \leq \beta$,

$$
\min \left(c_{1}, c_{2}, \ldots, c_{n}\right) \leq \operatorname{IVHFLTPWGHM}^{p, q, r}\left(c_{1}, c_{2}, \ldots, c_{n}\right) \leq \max \left(c_{1}, c_{2}, \ldots, c_{n}\right)
$$




\section{MADM with IVHFLTPWHM and IVHFLTPWGHM}

Here, we propose a MADM method based on the operators called IVHFLTPWHM and IVHFLTPWGHM. Alternatives and attributes are denoted by $Y=\left\{y_{1}, y_{2}, \ldots, y_{m}\right\}$ and $G=\left\{g_{1}, g_{2}, \ldots, g_{n}\right\}$, respectively, for a MADM question. Also, $w=\left(w_{1}, w_{2}, \ldots, w_{n}\right)^{T}$ denotes weights satisfying $w_{j} \in[0,1], \sum_{j=1}^{n} w_{j}=1$. Individual attribute preferences are given by each decision maker for different options pertinent to the prespecified set $S=\left\{S_{i} \mid i=0,1,2, \ldots, 2 t\right\}$. In the decision-making process, if several interval values are anonymously provided for alternatives $y_{i}$, concerning the variable $g_{j}$, such values could be regarded as an IVHFLE $y_{i j}$. In other cases, if the equal assessments are assigned by two evaluators, it appears just once in the IVHFLE $y_{i j}$. Suppose the decision matrix (DM) is denoted by $\mathbf{Y}=\left(y_{i j}\right)_{m \times n}$, where $y_{i j}$ designates the value of the alternative $y_{i}$ concerning the variable $g_{j}$ gauged by an IVHFLE.

Figure 1 shows a flowchart of the proposed approach. The optimal candidates could be reached by the following.

Step 1. Creation and normalization of the DM.

The DM is produced based on the evaluation of the attributes for each candidate. MADM problem generally includes the benefit and cost types of selection attributes. Even though benefit-type attributes need no normalization, cost-type needs it. Converting them could be done as follows:

$$
\tilde{y}_{i j}=\left\{\begin{array}{ll}
y_{i j,} & \text { for benefit attribute } g_{j}, \\
\operatorname{neg}\left(y_{i j}\right), & \text { for cost attribute } g_{j},
\end{array}, \quad(i=1,2, \ldots, m, \ldots j=1,2, \ldots, n) .\right.
$$

Thus, the normalized DM $\widetilde{\mathbf{Y}}=\left(\tilde{y}_{i j}\right)_{m \times n}$ can be obtained

Utilize the IVHFLTPWHM operator by equation (73).

Step 2. Obtaining the overall performance values.

$$
\begin{aligned}
& r_{i}=\operatorname{IVHFLTPWHM}^{p, q, r}\left(\tilde{y}_{i 1}, \tilde{y}_{i 2}, \ldots, \tilde{y}_{\text {in }}\right)
\end{aligned}
$$

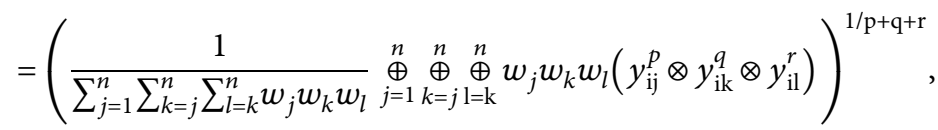

or the IVHFLTPWGHM operator

$$
\begin{aligned}
& r_{i}=\operatorname{IVHFLTPWGHM}^{p, q, r}\left(\tilde{y}_{i 1}, \tilde{y}_{i 2}, \ldots, \tilde{y}_{\text {in }}\right)
\end{aligned}
$$

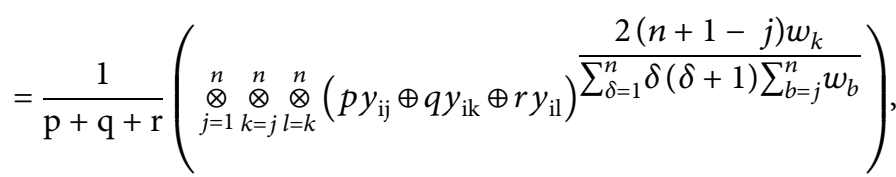

to obtain the collective scores $r_{i}(i=1,2, \ldots, m)$, for $p, q, r>0$.

Step 3. Finding the general scores.

Equation (8) helps compute scores expressed as $s\left(y_{i}\right)(i=1,2, \cdots, m)$ based on the overall performance values of $y_{i}$.

Step 4. Rating all options.

All candidates, $y_{i}(i=1,2, \ldots, m)$, are arranged according to the results of Step 3 following $s\left(y_{i}\right)(i=1,2, \ldots, m)$, and select the best one(s).

\section{Practical Examples}

This section provides a practical example which is given based on the proposed approach [28].

The ABC company represents a large government-run company specializing mainly in the production and sales of nonferrous metals in China. The company always seeks overseas investments to grow its key business. The department dealing with foreign investments has recently agreed upon utilizing preliminary surveys to generate alternatives based on several countries. After a detailed analysis, the 


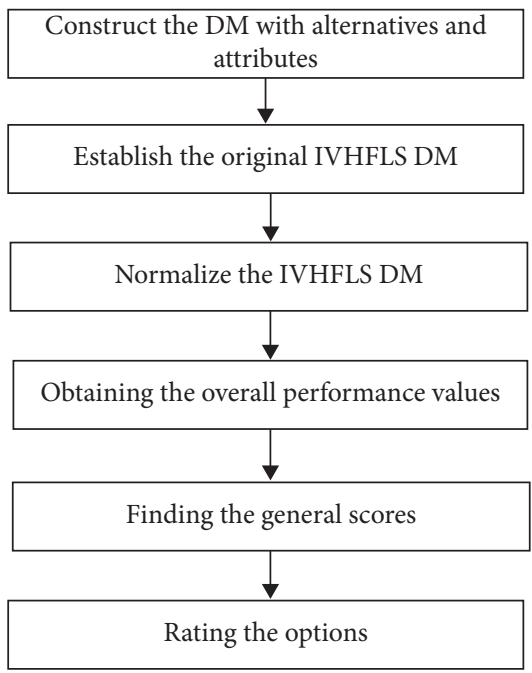

Figure 1: A flowchart of the proposed MADM method with HM operators.

department considered five alternatives $\left\{y_{1}, y_{2}, y_{3}, y_{4}, y_{5}\right\}$. Four factors were investigated according to the personnel experience in the department. These factors are $g_{1}$ : resources; $g_{2}$ : politics and policy; $g_{3}$ : economy; and $g_{4}$ : infrastructure. The decision information was assessed by $w=(0.1,0.3,0.2,0.4)^{T}$. The differences among the evaluators related to opinions could be clarified. Table 1 depicts decision information implemented by the IVHFLEs .

\subsection{Evaluation Based on IVHFLTPWHM or IVHFLTPWGHM Operators}

Step 1. The DM is constructed and normalization is conducted. Assume that no normalization is needed because all variables are benefit-type.

Step 2. For each alternative $y_{i}(i=1,2,3,4,5)$, general evaluation scores $r_{i}$ are combined by the IVHFLTPWHM and IVHFLTPWGHM operators according to equations (32) and (33) (see Tables 2 and 3 , and let $p=q=r=1$ ).

Step 3. Equation (8) helps compute the scores $s\left(y_{i}\right)$ of the collective performance values $y_{i}$ (see Table 4 ).

Step 4. The results of Step 3 help sort out the whole set of options $y_{i}$ (see Table 5).

As shown in Table 5, the alternative ranking results changed slightly. However, all methods agreed that $y_{5}$ is the best option, while the worst option is $y_{2} y_{4}$.

6.2. Comparison. When both rationality and superiority are a concern, the proposed methods presented in this paper are compared with the methods of Wang et al. [28] who introduced (IVHFLPWA) and (IVHFLPWG) operators. Comparisons were also performed against the method utilized by Gong et al. [29]. Moreover, the suggested distance measures and the aggregation operators are expanded to an IVHFL environment. Table 6 depicts the overall values $y_{i}$.

The results of the comparative analysis can be discussed as follows.
(1) Wang's [28] methods utilize the power average operator (PA) operator, and thus the attribute interrelations are not accounted for when the corresponding attribute values are aggregated. In brief, Wang's [28] approach assumes the independence of the attributes, but this is inconsistent with reality. The proposed aggregation operators in this paper are useful and meaningful and can capture the correlations of the aggregated arguments more proficiently when compared to the technique presented in Wang [28].

(2) Gong's [29] methods depend on the proposed distance measures for IVHFLSs. Indeed, the distance measurement method is realized by a comparison of the values. Also, Gong's [29] methods do not consider the interrelationship between attributes, and hence this method is not close to reality. In general, the proposed methods not only consider the interrelation between attributes but also extend the input variables to three parameters that can capture more decision-making information than techniques based on the existing HM operators. Analysis pertinent to efficacy and viability of investigated methodology is conducted.

6.3. Parameter Influence on Decision Making. When different values of parameters $p, q$, and $r$ in Step 1 are used to calculate the comprehensive evaluation values, the alternatives are rearranged as shown in Table 7 . It is seen from Table 7 that slight modifications are seen in ranking results. It is obvious that $y_{5}$ or $y_{3}$ is the best option. Then, $p, q$, and $r$ can be chosen according to the actual decision-making situation. However, the use of larger or smaller parameter values in the operators can make the calculations more complex and counter-intuitive as shown by Table 7 . Therefore, we generally recommend setting the values of the three parameters to unity. This shall make the calculations easier and also fully capture the correlation among aggregated arguments. 
TABle 1: The decision matrix for IVHFLS.

\begin{tabular}{ccccc}
\hline & $g_{1}$ & $g_{2}$ & $g_{3}$ & $g_{4}$ \\
\hline$y_{1}$ & $\left\langle s_{4},\{[0.3,0.5]\}\right\rangle$ & $\left\langle s_{5},\{[0.2,0.3],[0.4,0.5]\}\right\rangle$ & $\left\langle s_{4},\{[0.5,0.6],[0.7,0.8]\}\right\rangle$ & $\left\langle s_{3},\{[0.4,0.5]\}\right\rangle$ \\
$y_{2}$ & $\left\langle s_{2},\{[0.2,0.3],[0.5,0.8]\}\right\rangle$ & $\left\langle s_{4},\{[0.3,0.4],[0.4,0.7]\}\right\rangle$ & $\left\langle s_{3},\{[0.6,0.8]\}\right\rangle$ & $\left\langle s_{4},\{[0.3,0.4],[0.5,0.6]\}\right\rangle$ \\
$y_{3}$ & $\left\langle s_{3},\{[0.3,0.4],[0.5,0.7]\}\right\rangle$ & $\left\langle s_{4},\{[0.3,0.5]\}\right\rangle$ & $\left\langle s_{6},\{[0.7,0.8],[0.8,0.9]\}\right\rangle$ & $\left\langle s_{2},\{[0.6,0.7]\}\right\rangle$ \\
$y_{4}$ & $\left\langle s_{2},\{[0.3,0.4],[0.4,0.5],[0.5,0.6]\}\right\rangle$ & $\left\langle s_{3},\{[0.5,0.7]\}\right\rangle$ & $\left\langle s_{2},\{[0.3,0.5],[0.6,0.8]\}\right\rangle$ & $\left\langle s_{2},\{[0.8,0.9]\}\right\rangle$ \\
$y_{5}$ & $\left\langle s_{2},\{[0.3,0.6],[0.7,0.9]\}\right\rangle$ & $\left\langle s_{5},\{[0.4,0.7]\}\right\rangle$ & $\left\langle s_{3},\{[0.5,0.7],[0.8,0.9]\}\right\rangle$ & $\left\langle s_{4},\{[0.7,0.8]\}\right\rangle$ \\
\hline
\end{tabular}

TABle 2: The IVHFLTPWHM-based aggregation results.

\begin{tabular}{cc}
\hline & Overall evaluation values \\
\hline$r_{1}$ & $\left\langle s_{15.58},\{[0.34,0.45],[0.38,0.49],[0.40,0.51],[0.44,0.56]\}\right\rangle$ \\
$r_{2}$ & $\left\langle s_{10.34},\{[0.34,0.46],[0.37,0.57],[0.38,0.54],[0.41,0.65],[0.45,0.61],[0.48,0.70],[0.42,0.54],[0.45,0.64]\}\right\rangle$ \\
$r_{3}$ & $\left\langle s_{12.77},\{[0.50,0.62],[0.51,0.66],[0.52,0.65],[0.54,0.68]\}\right\rangle$ \\
$r_{4}$ & $\left\langle s_{7.47},\{[0.61,0.75],[0.64,0.79],[0.62,0.76],[0.65,0.77],[0.62,0.80],[0.65,0.80]\}\right\rangle$ \\
$r_{5}$ & $\left\langle s_{13.75},\{[0.54,0.70],[0.59,0.74],[0.58,0.74],[0.63,0.78]\}\right\rangle$ \\
\hline
\end{tabular}

TABLE 3: The IVHFLTPWGHM-based aggregation results.

\begin{tabular}{cc}
\hline & IVHFLTPWGHM-based aggregation results \\
\hline$r_{1}$ & $\left\langle s_{3.31},\{[0.43,0.57],[0.49,0.62],[0.46,0.59],[0.53,0.65]\}\right\rangle$ \\
$r_{2}$ & $\left\langle s_{2.71},\{[0.41,0.54],[0.48,0.64],[0.44,0.61],[0.51,0.70],[0.50,0.62],[0.57,0.71],[0.53,0.69],[0.60,0.76]\}\right\rangle$ \\
$r_{3}$ & $\left\langle s_{2.98},\{[0.57,0.70],[0.61,0.76],[0.58,0.71],[0.62,0.77]\}\right\rangle$ \\
$r_{4}$ & $\left\langle s_{1.94},\{[0.60,0.74],[0.63,0.76],[0.65,0.78],[0.67,0.80],[0.70,0.82],[0.72,0.84]\}\right\rangle$ \\
$r_{5}$ & $\left\langle s_{3.52},\{[0.60,0.79],[0.69,0.84],[0.64,0.81],[0.73,0.86]\}\right\rangle$ \\
\hline
\end{tabular}

TABLE 4: The score values associated with the alternatives.

\begin{tabular}{lcc}
\hline & IVHFLTPWHM & IVHFLTPWGHM \\
\hline$s\left(y_{1}\right)$ & 6.0831 & 1.7999 \\
$s\left(y_{2}\right)$ & 5.1794 & 1.5730 \\
$s\left(y_{3}\right)$ & 7.4629 & 1.9826 \\
$s\left(y_{4}\right)$ & 5.2301 & 1.4092 \\
$s\left(y_{5}\right)$ & $\mathrm{s}$ & 2.6144 \\
\hline
\end{tabular}

TABLE 5: Ranking results of the alternatives.

\begin{tabular}{lr}
\hline & \multicolumn{1}{c}{ Ranking } \\
\hline IVHFLTPWHM & $y_{5}>y_{3}>y_{1}>y_{4}>y_{2}$ \\
IVHFLTPWGHM & $y_{5}>y_{3}>y_{1}>y_{2}>y_{4}$ \\
\hline
\end{tabular}

TABLE 6: Overall values and ranking information of different approaches.

\begin{tabular}{|c|c|c|}
\hline Methods & Scores & Ranking results \\
\hline $\begin{array}{l}\text { Wang et al.'s method [35] by IVHFLPWA } \\
\text { operator }\end{array}$ & $\begin{array}{c}s\left(y_{1}\right)=0.280, s\left(y_{2}\right)=0.178, s\left(y_{3}\right)=0.268, s\left(y_{4}\right)=0.171 \\
s\left(y_{5}\right)=0.471\end{array}$ & $y_{5}>y_{3}>y_{1}>$ \\
\hline $\begin{array}{l}\text { Wang et al.'s method [35] by IVHFLPWG } \\
\text { operator }\end{array}$ & $\begin{array}{c}s\left(y_{1}\right)=0.275, s\left(y_{2}\right)=0.167, s\left(y_{3}\right)=0.254, s\left(y_{4}\right)=0.165 \\
s\left(y_{5}\right)=0.461\end{array}$ & $y_{5}>y_{3}>y_{1}>y_{2}>y_{4}$ \\
\hline Gong's method [36] based on & $\begin{array}{c}s\left(y_{1}\right)=0.209, s\left(y_{2}\right)=0.136, s\left(y_{3}\right)=0.324, s\left(y_{4}\right)=0.135 \\
s\left(y_{5}\right)=0.562\end{array}$ & $y_{5}>y_{3}>y_{1}>y_{2}>y_{4}$ \\
\hline $\begin{array}{l}\text { The proposed method by IVHFLTPWHM } \\
\text { operator }\end{array}$ & $\begin{aligned} s\left(y_{1}\right)=6.803, s\left(y_{2}\right)= & 5.179, s\left(y_{3}\right)=7.463, s\left(y_{4}\right)=5.230 \\
& s\left(y_{5}\right)=9.137\end{aligned}$ & $y_{5}>y_{3}>y_{1}>y_{4}>y_{2}$ \\
\hline $\begin{array}{l}\text { The proposed method by IVHFLTPWGHM } \\
\text { operator }\end{array}$ & $\begin{array}{c}s\left(y_{1}\right)=1.800, s\left(y_{2}\right)=1.573, s\left(y_{3}\right)=1.983, s\left(y_{4}\right)=1.409 \\
s\left(y_{5}\right)=2.614\end{array}$ & $y_{5}>y_{3}>y_{1}>y_{4}>y_{2}$ \\
\hline
\end{tabular}


TABLE 7: Ranking order by several values of $p, q$, and $r$ based on the IVHFLTPWHM operator.

\begin{tabular}{lcccccc}
\hline$p, q, r$ & $s\left(y_{1}\right)$ & $s\left(y_{2}\right)$ & $s\left(y_{3}\right)$ & $s\left(y_{4}\right)$ & $s\left(y_{5}\right)$ & Ranking results \\
\hline $1,1,2$ & 4.5092 & 4.6961 & 5.1225 & 3.7449 & 6.3960 & $y_{5}>y_{3}>y_{1}>y_{2}>y_{4}$ \\
$1,2,1$ & 6.5488 & 6.5974 & 7.7902 & 4.6665 & 9.3303 & $y_{5}>y_{3}>y_{2}>y_{1}>y_{4}$ \\
$1,2,2$ & 13.2644 & 12.2411 & 18.9705 & 6.8990 & 18.6342 & $y_{5}>y_{3}>y_{1}>y_{2}>y_{4}$ \\
$2,1,1$ & 9.8529 & 9.1763 & 11.6373 & 5.8280 & 13.4952 & $y_{5}>y_{3}>y_{1}>y_{2}>y_{4}$ \\
$2,1,2$ & 8.5569 & 7.9233 & 9.8943 & 5.2947 & 12.2065 & $y_{5}>y_{3}>y_{1}>y_{2}>y_{4}$ \\
$2,2,2$ & 22.5747 & 16.7440 & 30.2925 & 8.6475 & 30.5780 & $y_{5}>y_{3}>y_{1}>y_{2}>y_{4}$ \\
$3,1,1$ & 6.4275 & 6.0154 & 6.9402 & 4.4424 & 9.2085 & $y_{5}>y_{3}>y_{1}>y_{2}>y_{4}$ \\
$3,1,2$ & 8.6641 & 7.9220 & 9.3470 & 5.2896 & 12.8332 & $y_{5}>y_{3}>y_{1}>y_{2}>y_{4}$ \\
$3,3,3$ & 39.2554 & 19.6850 & 51.8694 & 9.5776 & 45.2869 & $y_{5}>y_{3}>y_{1}>y_{2}>y_{4}$ \\
$4,4,4$ & 283.3705 & 62.1517 & 401.9598 & 20.4737 & 305.4695 & $y_{5}>y_{3}>y_{1}>y_{2}>y_{4}$ \\
\hline
\end{tabular}

\section{Conclusions}

The HM operator is a very good tool to aggregate fuzzy information, and IVHFLSs exactly describe real situations of human hesitancy and uncertainty. In this work, we firstly propose novel HM operators, which can consider ternary or higher-order correlations among arguments and also satisfy the properties of reducibility and idempotency. Two of these operators are the three-parameter weighted HM (TPWHM) and the three-parameter weighted geometric HM (TPWGHM). We have generalized the novel HM operators within an IVHFL framework. Then, a numerical example pertinent to overseas investments is discussed [28]. The proposed approach is studied with detailed implementation and feasibility in addressing MADM problems. The presented approach is feasible and effective, considers correlations of any three arguments, and has some desirable properties. Finally, the presented method not only captures the correlations of the aggregated arguments, but also has demonstrated desirable performance by sensitivity and comparative analysis.

Generally, the decision matrix is excessively dependent on the decision makers. The presented methods do not show how the experts choose the overseas operators from the proposed ones and the associated interactions. We plan to apply the proposed methods to other research areas such as integration with probabilistic dual hesitant values, PFS, HPFS, and CHFS. Besides, it would be extended to other application domains such as machine learning, clustering analysis, performance evaluation, and so on as future research.

\section{Data Availability}

The data used to support the findings of this study are included within the article.

\section{Ethical Approval}

This article does not contain any studies with human participants or animals performed by any of the authors.

\section{Conflicts of Interest}

The authors declare that they have no conflicts of interest regarding the publication of this paper.

\section{Acknowledgments}

This study was supported by PhD Start-Up Fund of Shandong Technology and Business University (no. BS202106).

\section{References}

[1] F. Xiao, "EFMCDM: evidential fuzzy multicriteria decision making based on belief entropy," IEEE Transactions on Fuzzy Systems, vol. 28, no. 7, pp. 1477-1491, 2020.

[2] X. Liu, Z. Wang, and S. Zhang, "Novel correlation coefficient between hesitant fuzzy sets with application to medical diagnosis," Expert Systems with Applications, vol. 183, p. 115293, 2021.

[3] X. Liu, Z. Wang, and S. Zhang, "An approach to probabilistic hesitant fuzzy risky multiattribute decision making with unknown probability information," International Journal of Intelligent Systems, vol. 36, pp. 1-27, 2021.

[4] L. A. Zadeh, "The concept of a linguistic variable and its application to approximate reasoning-I," Information Sciences, vol. 8, no. 3, pp. 199-249, 1975.

[5] H. Bustince and P. Burillo, "Vague sets are intuitionistic fuzzy sets," Fuzzy Sets and Systems, vol. 79, no. 3, pp. 403-405, 1996.

[6] K. T. Atanassov, "More on intuitionistic fuzzy sets," Fuzzy Sets and Systems, vol. 33, no. 1, pp. 37-45, 1989.

[7] D. Joshi and S. Kumar, "Interval-valued intuitionistic hesitant fuzzy Choquet integral based TOPSIS method for multicriteria group decision making," European Journal of Operational Research, vol. 248, no. 1, pp. 183-191, 2016.

[8] X.-Y. Zou, S.-M. Chen, and K.-Y. Fan, "Multiattribute decision making using probability density functions and transformed decision matrices in interval-valued intuitionistic fuzzy environments," Information Sciences, vol. 543, pp. 410-425, 2021.

[9] G. Beliakov, S. James, J. Mordelová, T. Rückschlossová, and R. R. Yager, "Generalized Bonferroni mean operators in multi-criteria aggregation," Fuzzy Sets and Systems, vol. 161, no. 17, pp. 2227-2242, 2010.

[10] V. Torra, "Hesitant fuzzy sets," International Journal of Intelligent Systems, vol. 25, no. 6, pp. 529-539, 2010.

[11] V. Torra and Y. Narukawa, "On hesitant fuzzy sets and decision," in Proceedings of the IEEE International Conference on Fuzzy Systems, Jeju Island, Korea, August 2009.

[12] B. Farhadinia, "A series of score functions for hesitant fuzzy sets," Information Sciences, vol. 277, pp. 102-110, 2014.

[13] B. Farhadinia, "Information measures for hesitant fuzzy sets and interval-valued hesitant fuzzy sets," Information Sciences, vol. 240, no. 1, pp. 129-144, 2013. 
[14] M. Xia and Z. Xu, "Hesitant fuzzy information aggregation in decision making," International Journal of Approximate Reasoning, vol. 52, no. 3, pp. 395-407, 2011.

[15] Z. Xu and M. Xia, "On distance and correlation measures of hesitant fuzzy information," International Journal of Intelligent Systems, vol. 26, no. 5, pp. 410-425, 2011.

[16] D.-H. Peng, C.-Y. Gao, and Z.-F. Gao, "Generalized hesitant fuzzy synergetic weighted distance measures and their application to multiple criteria decision-making," Applied Mathematical Modelling, vol. 37, no. 8, pp. 5837-5850, 2013.

[17] Z. Xu and M. Xia, "Hesitant fuzzy entropy and cross-entropy and their use in multiattribute decision-making," International Journal of Intelligent Systems, vol. 27, no. 9, pp. 799822, 2012.

[18] Z. Xu and X. Zhang, "Hesitant fuzzy multi-attribute decision making based on TOPSIS with incomplete weight information," Knowledge-Based Systems, vol. 52, no. 6, pp. 53-64, 2013.

[19] M. Akram, S. Naz, and F. Smarandache, "Generalization of maximizing deviation and TOPSIS method for MADM in a simplified neutrosophic hesitant fuzzy environment," Symmetry, vol. 11, 2019.

[20] B. Zhu, Z. Xu, and M. Xia, "Hesitant fuzzy geometric Bonferroni means," Information Sciences, vol. 205, pp. 72-85, 2012.

[21] N. Chen, Z. Xu, and M. Xia, "Interval-valued hesitant preference relations and their applications to group decision making," Knowledge-Based Systems, vol. 37, no. 2, pp. 528-540, 2013.

[22] G. Wei, X. Zhao, and R. Lin, "Some hesitant interval-valued fuzzy aggregation operators and their applications to multiple attribute decision making," Knowledge-Based Systems, vol. 46, no. 4, pp. 43-53, 2013.

[23] D.-H. Peng, T.-D. Wang, C.-Y. Gao, and H. Wang, "Continuous hesitant fuzzy aggregation operators and their application to decision making under interval-valued hesitant fuzzy setting," Science World Journal, vol. 2014, Article ID 897304, 20 pages, 2014.

[24] S. Naz, M. Akram, and S. Alsulami, "Decision-making analysis under interval-valued q-Rung orthopair dual hesitant fuzzy environment," International Journal of Computational Intelligence Systems, vol. 14, no. 1, pp. 332-357, 2021.

[25] R. M. Rodríguez, L. Martínez, and F. Herrera, "A group decision making model dealing with comparative linguistic expressions based on hesitant fuzzy linguistic term sets," Information Sciences, vol. 241, pp. 28-42, 2013.

[26] Z. Xu, "Uncertain linguistic aggregation operators based approach to multiple attribute group decision making under uncertain linguistic environment," Information Sciences, vol. 168, 2004.

[27] R. Lin, X. Zhao, and G. Wei, "Models for selecting an ERP system with hesitant fuzzy linguistic information," Journal of Intelligent and Fuzzy Systems, vol. 26, no. 5, pp. 2155-2165, 2014.

[28] J.-Q. Wang, J.-T. Wu, J. Wang, H.-Y. Zhang, and X.-H. Chen, "Interval-valued hesitant fuzzy linguistic sets and their applications in multi-criteria decision-making problems," Information Sciences, vol. 288, pp. 55-72, 2014.

[29] R. Gong, W. Zhang, and D. Liu, "Multi-attribute decisionmaking method based on interval-valued hesitant fuzzy linguistic evaluation and its application," Statistics \& Decisions, vol. 35, no. 7, pp. 37-41, 2019.
[30] F. Meng, C. Wang, and X. Chen, "Linguistic interval hesitant fuzzy sets and their application in decision making," Cognitive Computation, vol. 8, no. 1, pp. 52-68, 2015.

[31] X.-B. Mao, S.-S. Hu, J.-Y. Dong, S.-P. Wan, and G.-L. Xu, "Multi-attribute group decision making based on cloud aggregation operators under interval-valued hesitant fuzzy linguistic environment," International Journal of Fuzzy Systems, vol. 20, no. 7, pp. 2273-2300, 2018.

[32] X. Feng, X. Shang, Y. Xu, and J. Wang, "A method to multiattribute decision-making based on interval-valued q-rung dual hesitant linguistic Maclaurin symmetric mean operators," Complex \& Intelligent Systems, vol. 6, no. 3, pp. 447-468, 2020.

[33] S. Skora, Mathematical Means and Averages: Generalized Heronian Means, 2009.

[34] G. Beliakov, A. Pradera, and T. Calvo, Aggregation Functions a Guide for Practitioners, 2007.

[35] C. Bonferroni, "Sulle medie multiple di potenze," Bollunmatital, vol. 5, pp. 267-270, 1950.

[36] D. Yu and Y. Wu, "Interval-valued intuitionistic fuzzy Heronian mean operators and their application in multicriteria decision making," African Journal of Business Management, vol. 6, pp. 4158-4168, 2012.

[37] G. Beliakov, A. Pradera, and T. Calvo, Aggregation Functions: A Guide for Practitioners, Springer, Berlin, Germany, 2007.

[38] H. Liu and D. Pei, "HOWA operator and its application to multi-attribute decision making," Journal of Zhejiang SCITECH University, vol. 25, pp. 138-142, 2012.

[39] D. Yu, "Intuitionistic fuzzy geometric Heronian mean aggregation operators," Applied Soft Computing, vol. 13, no. 2, pp. 1235-1246, 2013.

[40] P. Liu, Z. Liu, and X. Zhang, "Some intuitionistic uncertain linguistic Heronian mean operators and their application to group decision making," Applied Mathematics and Computation, vol. 230, no. 12, pp. 570-586, 2014.

[41] P. Liu, Q. Khan, and T. Mahmood, "Multiple attribute group decision making based on 2-tuple linguistic neutrosophic dombi Power Heronian mean operators," IEEE Access, vol. 99, pp. 1-23, 2019.

[42] L. Xu, Y. Liu, and H. Liu, "Linguistic interval-valued intuitionistic fuzzy copula Heronian mean operators for multiattribute group decision-making," Journal of Mathematics, vol. 2020, Article ID 6179468, 25 pages, 2020.

[43] S. Ayub, S. Abdullah, F. Ghani, M. Qiyas, and M. Yaqub Khan, "Cubic fuzzy Heronian mean Dombi aggregation operators and their application on multi-attribute decision-making problem," Soft Computing, vol. 25, no. 6, pp. 4175-4189, 2021.

[44] M. Lin, X. Li, and R. Chen, "Picture fuzzy interactional partitioned Heronian mean aggregation operators: an application to MADM process," Artificial Intelligence Review, vol. 225, 2021.

[45] C. Tian, J. Peng, and Z. Zhang, "A multi-criteria decisionmaking method based on single-valued neutrosophic partitioned Heronian mean operator," Mathematics, vol. 8, 2020.

[46] Z. S. Xu and Q. L. Da, "The uncertain OWA operator," International Journal of Intelligent Systems, vol. 17, no. 6, pp. 569-575, 2002.

[47] C. Na, Z. Xu, and M. Xia, "Interval-valued hesitant preference relations and their applications to group decision making," Knowledge-Based Systems, vol. 37, pp. 528-540, 2013. 